\title{
Lotic Ecosystem Health Assessments Using an Integrated Analytical Approach of Physical Habitat, Chemical Water Quality, and Fish Multi-Metric Health Metrics
}

\author{
Sang-Jae Lee ${ }^{1}$, Eui-Haeng Lee ${ }^{2}$, Kwang-Guk An ${ }^{1 *}$ \\ ${ }^{1}$ Department of Bioscience and Biotechnology, Chungnam National University, Daejeon, South Korea \\ ${ }^{2}$ Eui-Haeng Lee, Fishing Village Development Office, \\ Korea Rural Community Corporation, Naju, South Korea
}

Received: 3 August 2017

Accepted: 27 September 2017

\begin{abstract}
This study evaluates integrative lotic ecosystem health using neural network modeling and principal component analysis of physical, chemical, and biological parameters in 33 streams and rivers of a large watershed. Water chemistry parameters were measured to detect chemical health, and physical habitat health was determined by a model of qualitative habitat evaluation index (QHEI). Also, biological health was determined by the multi-metric community fish model of index of biological integrity (IBI) and then analyzed trophic compositions and tolerance guilds. In addition, we analyzed fish tissues of liver, kidney, gill, vertebra, and muscle using a sentinel species of Zacco platypus. Chemical pollutions were closely associated with land-use patterns within the watershed and the locations of major point-sources. Model value of QHEI as a measure of physical habitat health averaged 144, indicating good health, and varied from 96 to 190 depending on the sampling sites. The proportion of sensitive fish species in the tolerance guilds had negative correlation with organic matter pollution $(\mathrm{r}=-0.716, \mathrm{p}<0.001)$ and had positive a relationship with IBI $(\mathrm{r}=0.683, \mathrm{p}<0.001)$ and QHEI $(\mathrm{r}=0.573, \mathrm{p}=0.001)$. The proportion of insectivore species, as a trophic composition indicator, was inversely correlated with BOD $(\mathrm{r}=-0.463, \mathrm{p}=0.007)$ and positive with IBI $(\mathrm{r}=0.679, \mathrm{p}<0.001)$. The analysis of the multi-layer perceptron (MLP) 14-5-1 model, based on the predicted IBI values in the training sites $\left(\mathrm{R}^{2}=0.999, \mathrm{MSE}=0.015\right)$ and testing sites $\left(\mathrm{R}^{2}=0.894, \mathrm{MSE}=27.4\right)$ showed high efficiency in the MLP model.
\end{abstract}

Keywords: lotic ecosystem health, integrated approach, water chemistry, biotic integrity, fish model

*e-mail:kgan@cnu.ac.kr 


\section{Introduction}

Lotic ecosystems in temperate regions have been largely impacted by various sources of organic matter pollutants and nutrients and physical habitat disturbances [1]. Especially effluents from industrial complexes and wastewater treatment plants are known to be a major source of chemical pollution in urban streams and rivers. Similarly, urban developments and expending residential areas modify habitat structures such as channel shapes [2], substrate composition, and flow regimes of stream ecosystems [3]. As a result, ecological degradations in regional streams are directly or indirectly influenced by chemical pollution alone or physical habitat disturbance alone, or the combined effects of the two [4]. Recent research [5] has pointed out that these degradations are closely associated with land use patterns of agricultural cropland, forest, or urban areas, and especially nutrient pollution by nitrogen and phosphorus are largely influenced by fertilizers used in croplands.

Stream research has so far been frequently diagnosed on the basis of conventional chemical measurements [6]. The chemical approach, however, could not reflect integrative effects from stream channelization, barriers, altered hydrological regimes, and all complex biological factors (exotic species) happening in stream ecosystems [7]. Most chemical approaches, based on chemical measurements, could detect specific types of degradation only associated with chemical sources like eutrophication of nitrogen and phosphorus or organic or inorganic toxicants, but did not reflect the habitat alterations of stream environments or biological health [8-9]. For these reasons, integrated approaches using biological, chemical, and physical parameters were required for efficient stream health monitoring and management [10-12].

One of the first stream health monitoring approaches was the index of biological integrity (IBI) concept, which was established as a multi-metric assessment of rapid bioassessment protocol (RBP) using various biota including fish for efficient stream management in the United States. Another approach is the water framework directive (WFD) developed in European communities using various multi-metric bioassessment models for effective management and conservation in European streams and rivers [13]. These models reflect changes of sensitive species [4], tolerant species, feeding guilds, morphological anomalies, and exotic species [14-16]. This approach is known as a biological integrity model using various trophic levels of biota. Recently in Korea, national stream health assessment methodologies using biota such as periphyton, macroinvertebrate, and fish [17-18] were developed to evaluate the lotic ecosystems (wadable streams and rivers) by the Ministry of Environment in Korea. The regional monitoring methodologies have been a key role to diagnose stream/ river health degradations in numerous other countries as well as Korean aquatic ecosystems [18]. It also provided national biological standards or criteria to manage and restore stream health impacted in the major watersheds in Korea. For successful management to keep watersheds clean, research should be conducted through comparative analysis between past and present health conditions of aquatic environments and also predict the future.

Regional model developments of the IBI are very important due to high continental or regional heterogeneities in species compositions, trophic guilds, and feeding types compared to the original models. For this reason, various regional models were developed in the USA [19], Canada [20], France [21], India [22], and China [23] to study lotic ecosystem health using fish assemblages that have been reported in the Geum River watershed [24]. However, little is known about integrative assessments of stream health. Chemical analysis such as nutrients, organic pollutants, and heavy metals have frequently been reported in the watershed [25-26]. In the meantime, some studies [27] have pointed out the importance of physical habitats as well as chemical conditions for stream ecosystem health. Recently, regional-scale studies of multi-metric fish models demonstrated metric attributes for model development, the model criteria, and their applications $[17,28]$, and the model was applied to urban streams, pristine forestry streams [28], and agricultural streams [9]. The model diagnosed the integrative health of stream ecosystems and thus provided management strategies for stream restorations and conservation in the watershed.

In this study, we evaluated integrative lotic ecosystem health using neural network modeling and principal component analysis, and physical, chemical, and biological parameters were analyzed at 33 streams and rivers of a large watershed. Water quality parameters were measured to detect chemical health, and physical habitat health was determined by a metric QHEI model. Also, biological health was determined by the multi-metric community fish model of index of biological integrity (IBI), and then analyzed trophic compositions and tolerance guilds. Integrated ecosystem health in the watershed was evaluated using chemical, physical habitat, and biological health analysis.

\section{Materials and Methods}

\section{Study Sites and Sampling Methods}

This study was conducted in the Geum River watershed, located in the temperate region of South Korea (Fig. 1). Fish sampling was conducted at 33 sites, including mainstream and tributaries in October 2007 according to the modified wading method [29]. We also sampled 39 reference streams and determined the stream order after the methodology of Strahler [30] using a map scale of $1: 120,000$. 


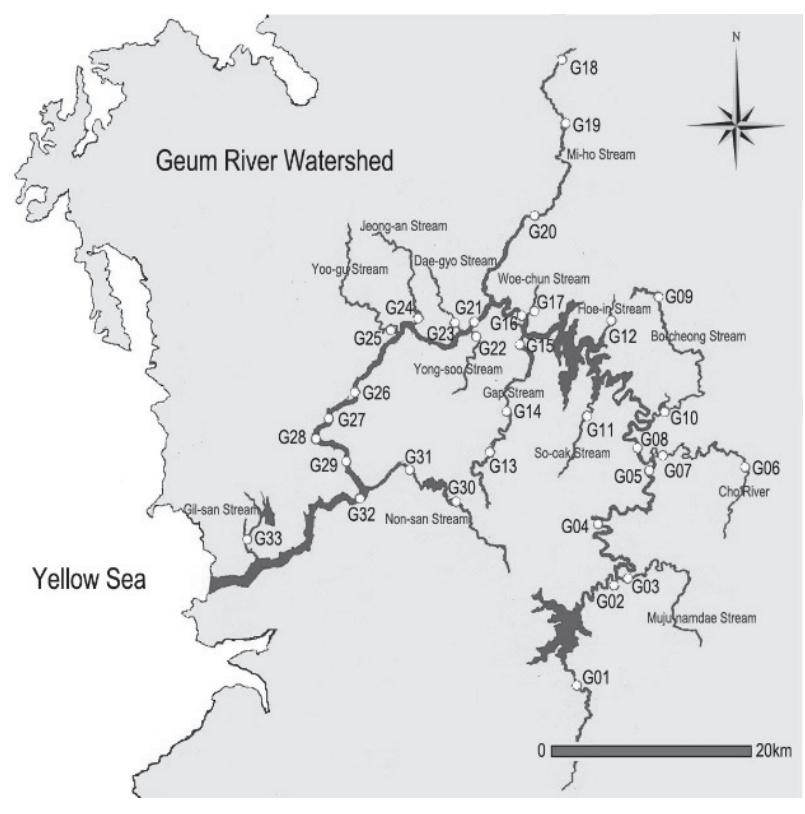

Fig. 1. Sampling sites (G) in the Geum River Watershed.

For fish sampling, all habitat types including riffle, run, and pool were sampled and sampled in an upstream direction for at least $200 \mathrm{~m}$ distance during 50 minutes respectively at each site for the catch per unit efforts (CPUE). Sampling gears were kick-net $(4 \times 4 \mathrm{~mm})$ and casting-net $(5 \times 5 \mathrm{~mm})$. All fish were identified at the sampling locations and then released, some ambiguous specimens to identify, however, were preserved in $10 \%$ formalin. All fish were examined for external characteristics (DELT; [31]) of deformities (D), erosion (E), lesion (L), and tumors (T). Tolerance and trophic guild analyses were based on previous regional studies.

\section{Chemical Water Quality Analysis}

Chemical water quality dataset during 20052007 were obtained from the National Water Quality Monitoring Program (NWQMP) of the Korean Ministry of Environment (MEK). The parameters used in the study were biological oxygen demand (BOD), total suspended solids (TSS), electric conductivity (EC), and total phosphorus (TP).

\section{Habitat Evaluation Model}

Habitat health was evaluated by the qualitative habitat evaluation index (QHEI), which was developed by Plafkin et al. [32]. We selected 11 habitat parameters based on the previous widely used references. For the habitat assessments we included primary, secondary, and tertiary components to the habitat model and the metrics are substrate structure and vegetation coverage, channel characteristics, bank characteristics, and bank structure. All metric characteristics were described in the previous research by An et al. [17]. The health conditions of the habitat were judged by the summation of scores obtained from the 11 parameters and were ranked as excellent (A, 182-220), good (B, 124-168), fair (C, 66-110), and poor (D, 8-52) conditions.

\section{Analysis of Total Mercury Concentrations in Fish Tissues}

Total mercury concentrations in various fish tissues such as liver, kidney, gill, vertebra, and muscle were analyzed by direct mercury analyzer (DMA-80, US EPA Method 7473), and the target fish was Zacco platypus. Method 7473 of US EPA [33] is known as a spectroscopic analytical method that has no pretreatment of samples and directly ignites at high temperatures. Samples of fish tissues were analyzed by the DMA-80 based on thermal decomposition, catalytic reduction, and amalgamation desorption. The fish tissues were initially dried in the oxygen stream passing through a quartz tube located inside a controlled heating coil under the condition of oxygen supplies as a carrier gas to each cylinder. The combustion gases were further decomposed on a catalytic column. Mercury vapor was collected on gold amalgamation traps and subsequently desorbed for a quantification. The mercury content was determined using a single-beam spectrophotometer with two sequential, flow-through measurement cells under the condition of $254 \mathrm{~nm}$. The light source for the spectrophotometer was a low-pressure mercury vapor lamp, and a silicon UV photo-detector was used for analysis.

\section{Biological Integrity Model}

Biological health was diagnosed by the 8-metric fish model based on the IBI, instead of the 12-metric model, originally suggested in U.S. Midwestern streams by Karr [15]. We modified the model for the regional application of the Geum River Watershed and developed the trophic and tolerance guilds classifications in this region. The metrics (M) were composed of three major groups such as ecological characteristic and species composition, trophic composition, and fish abundance and health condition. Metric scores of 1, 3, or 5 were assigned to each of the raw metric values after the RBP approach of the U.S. EPA. Each metric and criteria characteristics have been described previously [17, 34]. These scores were then summed to obtain a site-specific model value that ranged from 8 to 40 , and categorized with four ranks (excellent, A, 36-40; good, B, 26-35; fair, C, 16-25; and poor, D, 8-15) to be used.

\section{Artificial Neural Network Modeling}

The model of an artificial neural network (ANN) is a good tool for recognizing efficient patterns with abstraction and simplification of complex non-linear physical and chemical data. This approach is known as one of the most effective methods for predicting ecological dynamics and variations [35]. We used 
Neuro Solution 5.07 for the model analysis and applied it to multilayer perceptron (MLP) analysis for the development of artificial neural network modeling.

We used various physical and chemical variables as the input layer and the values of IBI model as the output layer (Table 1, Fig. 2). Among all 33 sampling sites, we applied 25 sites as a training test to learn the model, and then 8 sites were determined as the testing sites to validate the ANN model. Neural network breadboard was determined by the Tan-Sigmoid function. For network training, epoch number was decided at 500 and activated 3 times repetitively, through iteration learning, so that we determined the MLP model with the most adaptive minimum mean squared error by \pm 1 standard deviation (Fig. 2). Dataset analysis using the neural network model was proceeded to come up with observed IBI and predicted IBI through the test by optimal weight from training and testing dataset, respectively. Also, sensitivity analysis between input and output variables was conducted by the training dataset.

Table 1. Input and output variables to the artificial neural network model.

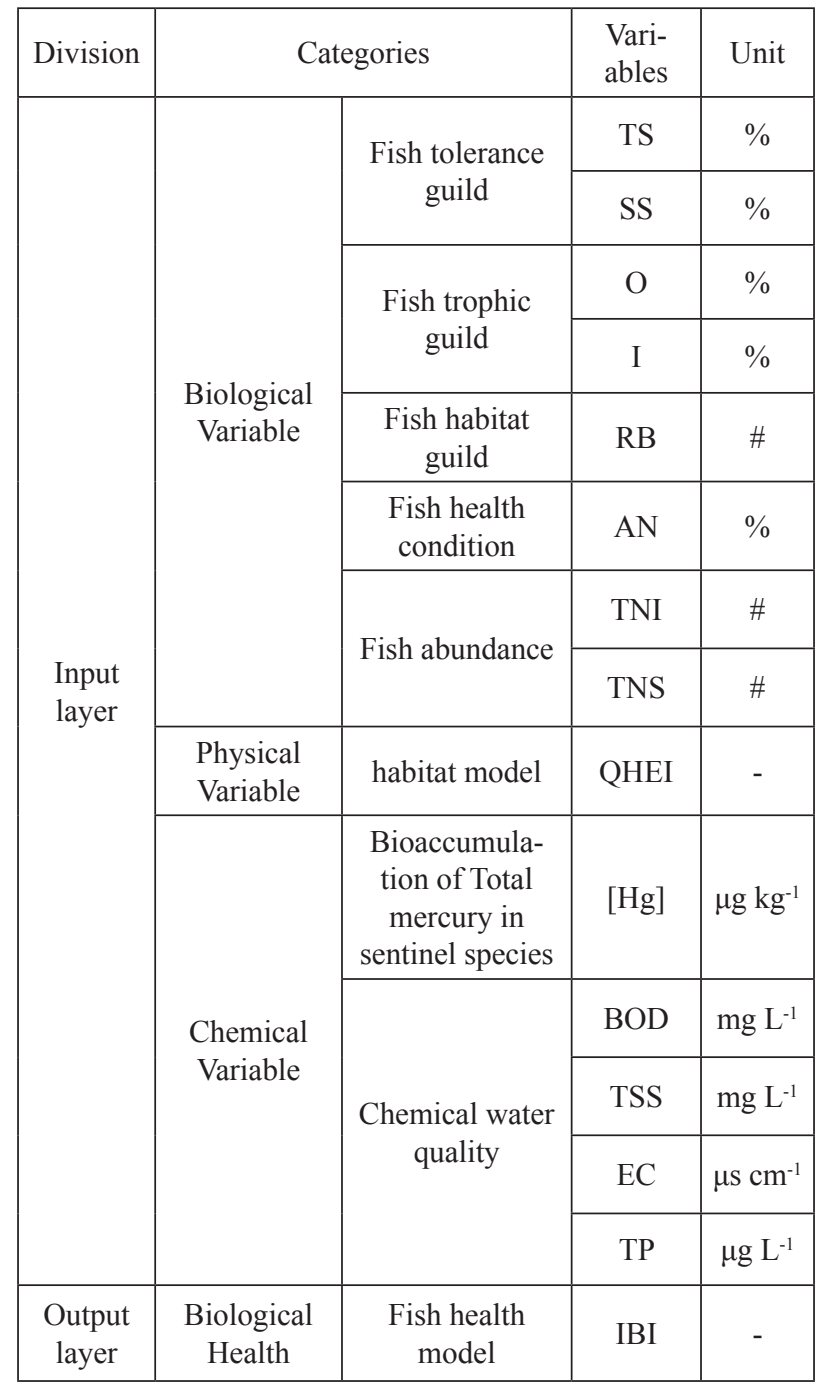

\section{Statistical Analysis}

The statistical analysis of SPSS (Version 12.0KO for windows; [36]) was used for correlation analysis, regression analysis, cluster analysis, and principal component analysis (PCA). Also, the program of PCOrd was simultaneously used for the PCA and cluster analysis. PCA was used to identify the primary gradients in the IBI, independent of the IBI metrics, QHEI metrics, total mercury of the sentinel species, and water quality parameters.

\section{Results and Discussions}

\section{Chemical Water Quality}

Data analysis of chemical water quality showed a large variation along the longitudinal gradients of upstream to downstream depending on the point-sources and the land-use pattern. Concentrations of BOD, as a measure of organic pollution, averaged $2.1 \mathrm{mg} \mathrm{L}^{-1}$, indicating good chemical health by the chemical criteria, but ranged between 0.1 and $12.9 \mathrm{mg} \mathrm{L}^{-1}(\mathrm{n}=1188$; Fig. 3). Values of BOD were $<1.0 \mathrm{mg} \mathrm{L}^{-1}$ in the upstream pristine regions but $>6.0 \mathrm{mg} \mathrm{L}^{-1}$ at some polluted sites (G15, G21) due to effluents from an industrial complex and wastewater treatment plants. The major polluted

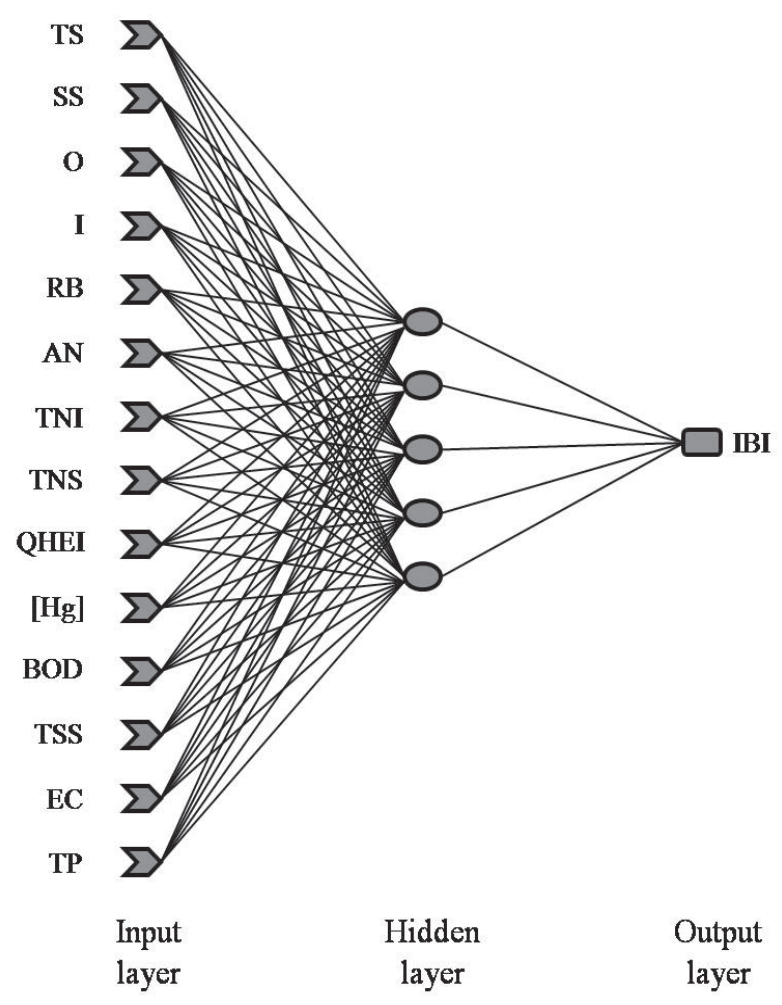

Fig. 2. Diagram of the multi-layer perceptron (MLP) in the 145-1 model. 

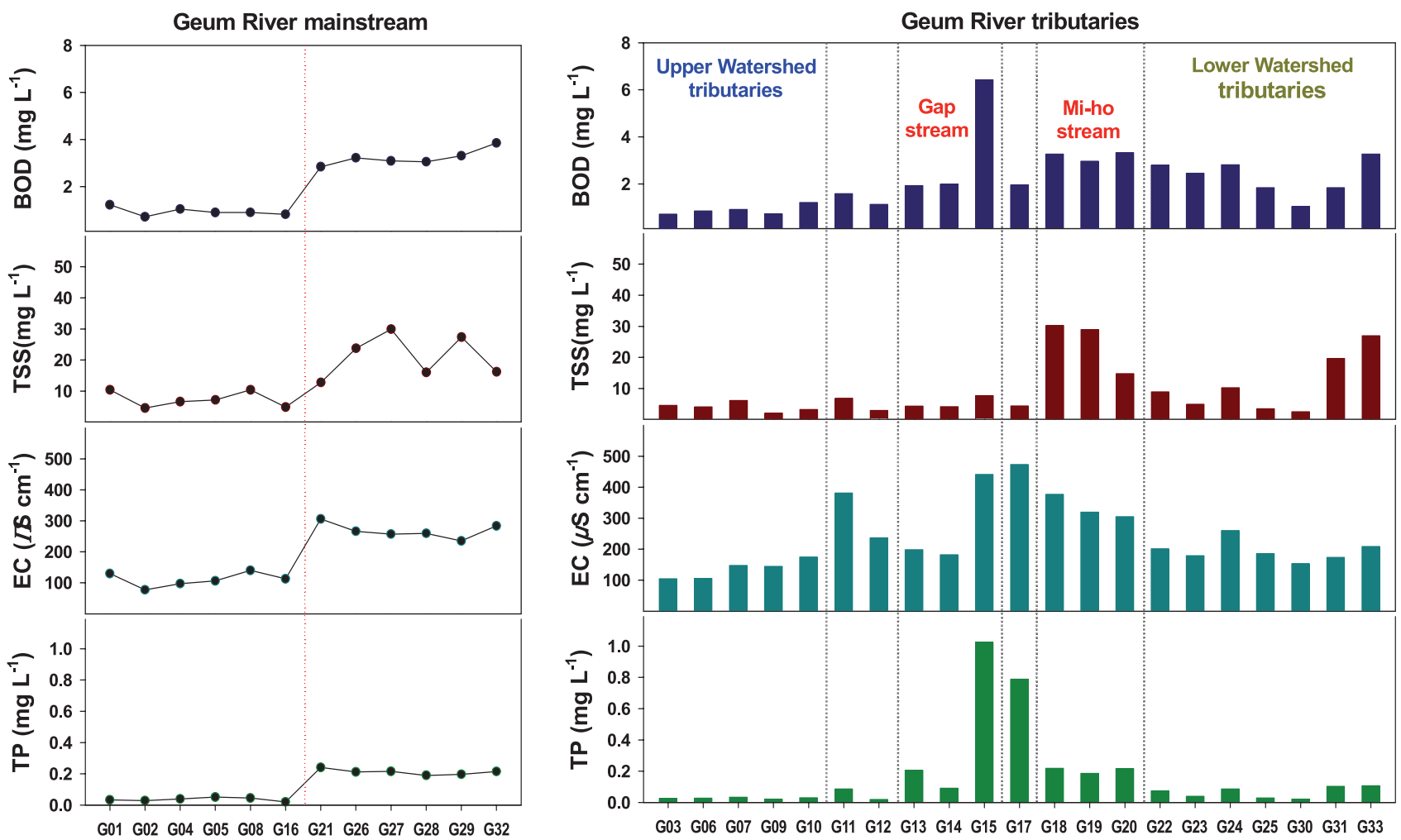

Fig. 3. Chemical water quality at the sampling sites of Geum River watershed.

streams were identified as Miho and Gap, and these sites were most pronounced in the nutrient pollution of total phosphorus (TP). Total suspended solids (TSS) averaged $11.2 \mathrm{mg} \mathrm{L}^{-1}$ (range: $0.1-472.0 \mathrm{mg} \mathrm{L}^{-1}$ ) in the watershed and were the highest in the upstream reach $(\mathrm{G} 18$, mean $30.3 \mathrm{mg} \mathrm{L}^{-1}$ ) and the midstream reach (G19, mean $\left.29.0 \mathrm{mg} \mathrm{L}^{-1}\right)$ of Miho Stream. The fluctuation pattern of suspended solids was similar to the BOD values in the sampling sites. Specific electrical conductivity (EC), as a measure of dissolved ions, averaged $219 \mu \mathrm{s} \mathrm{cm}^{-1}$, and the high values were affected by the effluents of industrial complex and agricultural farms. Total phosphorus (TP), as an indicator of trophic state averaged $0.149 \mathrm{mg} \mathrm{L}^{-1}$ and ranged between 0.001 and $3.216 \mathrm{mg} \mathrm{L}^{-1}$ depending on the locations of the point and non-point sources. Values of TP were low in the pristine upstreams, and the variation pattern when TP values were $<0.5 \mathrm{mg} \mathrm{L}^{-1}$ was similar to the $\mathrm{BOD}$, indicating that organic pollution occurred in the nutrient-polluted sites.

\section{Physical Habitat Health}

Multi-metric model values of the qualitative habitat evaluation index (QHEI) were estimated by a summation of metrics from in-stream substrate type, flow condition, channel modification, and bank characteristics (Table 2). During the study, QHEI values averaged 144 ( $\mathrm{n}=$ 33) and ranged between 96 and 190, indicating a good physical habitat health condition according to habitat criteria [19]. But habitat health tended to be degrading slowly from upstream to downstream. The value of
QHEI in mainstream averaged $155(\mathrm{n}=12)$ in all the streams and $174(\mathrm{n}=6)$ in the headwater streams with excellent-good condition, indicating that physical habitat was maintained well in the headwater region. The QHEI values in the G21-G32, however, averaged $120(\mathrm{n}=6)$, indicating a good-fair condition with minor habitat disturbance. Particularly the downstream site of G32 showed severe habitat simplification derived from sedimentations of silt and organic matters due to park constructions for human activities.

In tributaries of the Geum River, mean QHEI was low $(138, \mathrm{n}=21)$, which was caused by habitat deterioration from non-point source pollutions. G03 (QHEI: 110) was maintained well in habitat condition but affected by disturbances from human activity such as weir and some recreational parks located along the stream. In Gap Stream (G13-G15), a typical urban stream, it was proceeded by organic sedimentation and disturbances occurring from residential effluents and industrial complex in G14 (QHEI: 101). In G17 (101) and G18 (96), it was simplified by sand for stream substrate and was affected by flow reduction related to seasonal factors. Relative Habitat Quality (RHQ) was applied using the QHEI model modified (Fig. 4). Analysis of primary habitat components (substrate structure and vegetation coverage) showed that sand sedimentation and habitat simplification resulted in greater impacts in the downstream than the upstream reach. Metric scores of H1 (substrate/instream cover, 66.5\%) and H2 (embeddedness, 65.8\%). H3 (flow velocity/depth, 76.1\%) were low in some streams along with simplified instream 


\begin{tabular}{|c|c|c|c|c|c|c|c|c|c|c|c|c|c|c|c|c|c|c|c|c|c|c|c|c|c|c|}
\hline & $\bar{U}$ & $\infty$ & $\infty$ & $\infty$ & $\stackrel{0}{-1}$ & 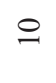 & $\infty$ & $\infty$ & r & iิ & $N$ & 6 & $\Xi 0$ & $\hat{\mathcal{S}}$ & in & $m$ & $\infty$ & 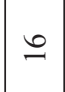 & $\cong$ & $\stackrel{\infty}{-}$ & in & $\stackrel{\infty}{-}$ & i & 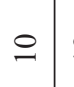 & $=\bar{a}$ & $\overline{\underline{m}} \widehat{\theta}$ \\
\hline & రే & $\because$ & $\because$ & $\because$ & 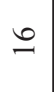 & $\cong$ & $\stackrel{\infty}{\sim}$ & 6 & $\because$ & $\stackrel{\infty}{\simeq}$ & $\stackrel{\bullet}{\longleftarrow}$ & ते & 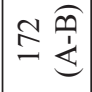 & हి & 0 & 0 & $\because$ & $\underline{0}$ & $\because$ & $\infty$ & 0 & 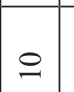 & 0 & 0 & $=\ddot{g}$ & $\cong 0$ \\
\hline & U & $=$ & $=$ & 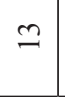 & $\stackrel{\infty}{=}$ & $\simeq$ & 0 & $\infty$ & $\simeq$ & $\infty$ & $\sim$ & $\infty$ & $\begin{array}{ll}\cong & 0 \\
\exists & \oplus\end{array}$ & $\overrightarrow{0}$ & त & i & $\propto$ & $\underline{0}$ & $\simeq$ & $\cong$ & 0 & $\stackrel{\infty}{\sim}$ & i & 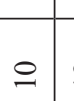 & $\cong$ & $\stackrel{8}{\underline{8}}$ \\
\hline & U & 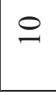 & $=$ & $=$ & $\stackrel{\infty}{=}$ & $\stackrel{\infty}{\simeq}$ & in & in & $\nabla$ & 0 & in & $\infty$ & $\Xi 0$ & 8 & 잉 & $\underline{\square}$ & ì & $\cong$ & $\circ$ & $\infty$ & 0 & 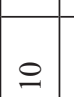 & 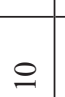 & $\sim$ & $6 ?$ & $\Xi 0$ \\
\hline & Jै & $\stackrel{0}{-}$ & $\cong$ & $\because$ & 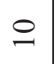 & $\infty$ & $\because$ & 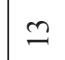 & ㅇ & $\stackrel{0}{-1}$ & 0 & in & $\bar{\Xi} \widehat{\otimes}$ & & & & & & & & & & & & & \\
\hline & & & & & & & & & & & & & & రิ & in & 0 & $\because$ & $\stackrel{\infty}{-\infty}$ & $\stackrel{\infty}{\sim}$ & $\stackrel{2}{2}$ & 0 & \pm & $\stackrel{\infty}{\stackrel{\infty}{2}}$ & $=$ & $\stackrel{\wedge}{\triangle}$ & I $\hat{\theta}$ \\
\hline & $\tilde{J}$ & 4 & $-\infty$ & $=1$ & $n$ & $\infty$ & $\cong$ & $\stackrel{\infty}{=}$ & $\infty$ & \pm & 9 & 0 & $\ddot{1} \tilde{\theta}$ & & & & & & & & & & & & & \\
\hline & $\bar{J}$ & $\stackrel{\infty}{\sim}$ & 0 & $\stackrel{\infty}{\sim}$ & 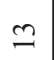 & $\cong$ & $\cong$ & $\cong$ & 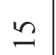 & $\stackrel{\infty}{\simeq}$ & $\simeq$ & $\infty$ & in $\widehat{\theta}$ & 0 & - & & & - & -1 & & & - & -1 & . & -7 & 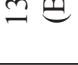 \\
\hline & & & & & & & & & & & & $n$ & & $\widehat{\widehat{\mho}}$ & in & $=$ & 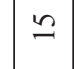 & $\cong$ & $\infty$ & $\stackrel{\infty}{\sim}$ & 0 & $\stackrel{\infty}{\simeq}$ & $\stackrel{\infty}{=}$ & $\simeq$ & $\simeq$ & ભેહિ \\
\hline & & & & & & & & & & & & & & $\sim$ & $\infty$ & $=$ & $n$ & $\infty$ & 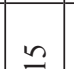 & $m$ & 0 & 0 & 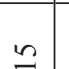 & $\therefore$ & 8 & toิ \\
\hline & छे & $\stackrel{\infty}{\sim}$ & $\because$ & त & 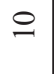 & $\infty$ & $\stackrel{\infty}{\stackrel{-1}{2}}$ & 9 & 2 & iి & $\because$ & $\simeq$ & $\cong \stackrel{m}{=}$ & 0 & & & & & & & & & & & & \\
\hline స్ & 我 & $\simeq$ & $\stackrel{-\infty}{-1}$ & $\because$ & 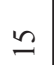 & $\cong$ & $\cong$ & $\infty$ & 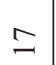 & $\stackrel{\infty}{\sim}$ & 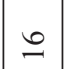 & $\cong$ & $\stackrel{\bullet}{0} \widehat{\theta}$ & సิ & $\stackrel{\infty}{-}$ & 9 & $\stackrel{\infty}{\simeq}$ & 9 & 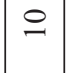 & $\stackrel{2}{2}$ & $\cong$ & \pm & 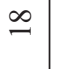 & $\stackrel{0}{0}$ & $\therefore 5$ & छิ \\
\hline & ì & ㄱ. & ¿ి & 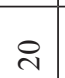 & $\cong$ & 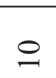 & $\cong$ & $\cong$ & \pm & \pm & $\cong$ & $\simeq$ & $\Xi \widehat{P}$ & J્ડ & 0 & $=$ & $\infty$ & $=$ & $\cong$ & $\cong$ & 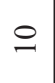 & $\simeq$ & 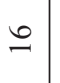 & 은 & ¿ి & $\underline{\underline{m}}$ \\
\hline & : & $\stackrel{\infty}{\sim}$ & 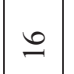 & $\stackrel{\infty}{-\infty}$ & $\approx$ & $=$ & $\cong$ & $\infty$ & $\stackrel{\infty}{1}$ & i & \pm & $\infty$ & î $\widehat{x}$ & శ్ర & $\infty$ & 6 & 9 & $\cong$ & 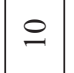 & $\because$ & $\stackrel{0}{0}$ & $\stackrel{\circ}{\circ}$ & 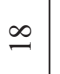 & $\stackrel{0}{0}$ & $=\varsubsetneqq$ & ఫิê \\
\hline & 管 & $\stackrel{\infty}{-}$ & 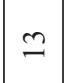 & $\cong$ & 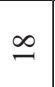 & $\stackrel{\infty}{\simeq}$ & $\because$ & 0 & $\stackrel{\infty}{-1}$ & i & $m$ & 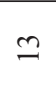 & $\ddot{\theta} \widehat{\theta} \widehat{\theta}$ & ปิ & 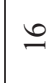 & $\cong$ & $\because$ & $\because$ & $=$ & 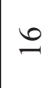 & $\infty$ & $\infty$ & ì & 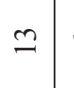 & $0 \%$ & 气ิ \\
\hline & 总 & i & $\stackrel{\infty}{\sim}$ & $\stackrel{\circ}{-1}$ & $\stackrel{0}{2}$ & $\cong$ & $=$ & 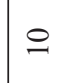 & 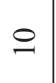 & I & $\underline{0}$ & $\cong$ & $\stackrel{\infty}{\circ} \widehat{\theta} \widehat{\theta}$ & $\overrightarrow{\widetilde{O}}$ & 9 & 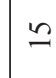 & $\cong$ & 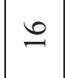 & $\simeq$ & 잉 & 0 & $\circ$ & 9 & \pm & $\cong g$ & ఫิ \\
\hline & ồ & $\because$ & 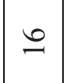 & 0 & $\stackrel{0}{-1}$ & $\cong$ & 0 & $\infty$ & $\nabla$ & $\sigma$ & 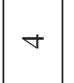 & 0 & $\stackrel{0}{0}$ & త్రి & 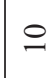 & 9 & 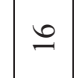 & 9 & $=$ & $\cong$ & 0 & 6 & 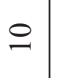 & $\stackrel{0}{\circ}$ & $=$ & $\widehat{\underline{y}} \widehat{\theta}$ \\
\hline & ठิ & $i$ & $\stackrel{\infty}{\sim}$ & $\stackrel{\sim}{\pi}$ & 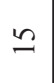 & 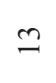 & $\stackrel{\infty}{\circ}$ & $\cong$ & त) & iి & ন & $=$ & 92 & डे & $=$ & $\stackrel{n}{\sim}$ & 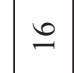 & $\because$ & $\cong$ & $\because$ & $\infty$ & $\stackrel{0}{\sim}$ & 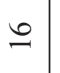 & \pm & $=9$ & 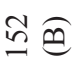 \\
\hline & $\overrightarrow{0}$ & $\stackrel{\infty}{\sim}$ & $\because$ & 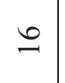 & $\stackrel{\infty}{-1}$ & $\cong$ & $\stackrel{\infty}{.}$ & $\cong$ & त & iి & $\stackrel{\circ}{\circ}$ & $\cong$ & $\infty$ & $\frac{\infty}{0}$ & in & $m$ & $\infty$ & 0 & $\infty$ & $\cong$ & $=$ & $\infty$ & $\cong$ & 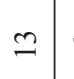 & 0 & $\approx 00$ \\
\hline & 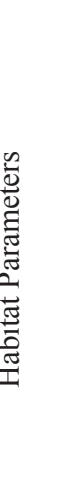 & 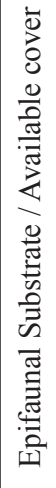 & 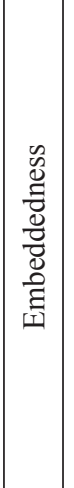 & 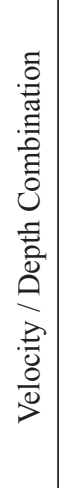 & 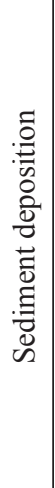 & 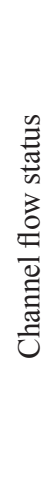 & 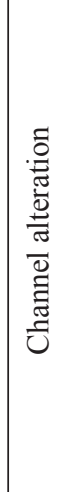 & 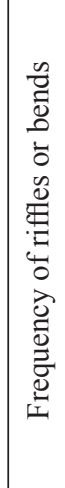 & 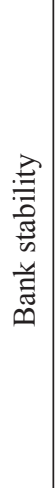 & 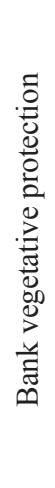 & 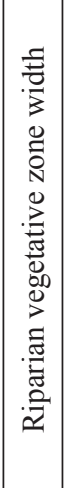 & 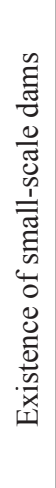 & 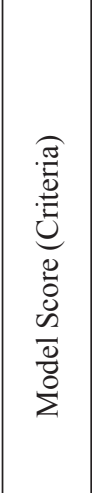 & & 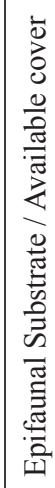 & 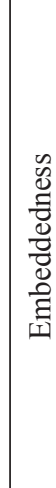 & 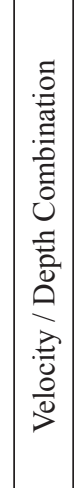 & 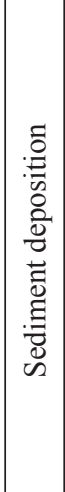 & 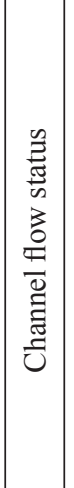 & 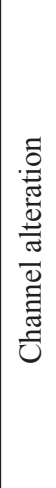 & 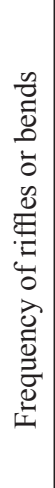 & 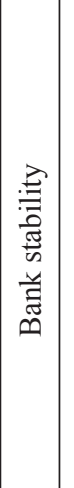 & 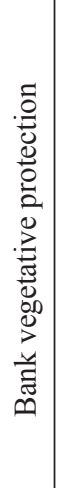 & 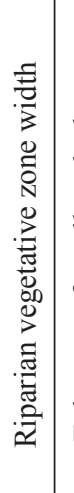 & 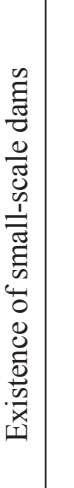 & 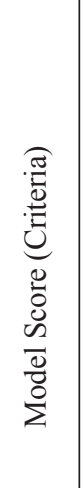 \\
\hline & & $\Xi$ & $\cong$ & $\ddot{M}$ & : & 声 & 12 & $\Xi$ & $\stackrel{\infty}{\infty}$ & શิ & $\stackrel{\ominus}{I}$ & $\bar{\Xi}$ & & & $\Xi$ & 조 & $\underline{\mathbf{I}}$ & $\mathbb{2}$ & \pm & 노 & 운 & \begin{tabular}{|l|} 
\\
\end{tabular} & शे & $\stackrel{O}{ \pm}$ & $\bar{\Xi}$ & \\
\hline
\end{tabular}




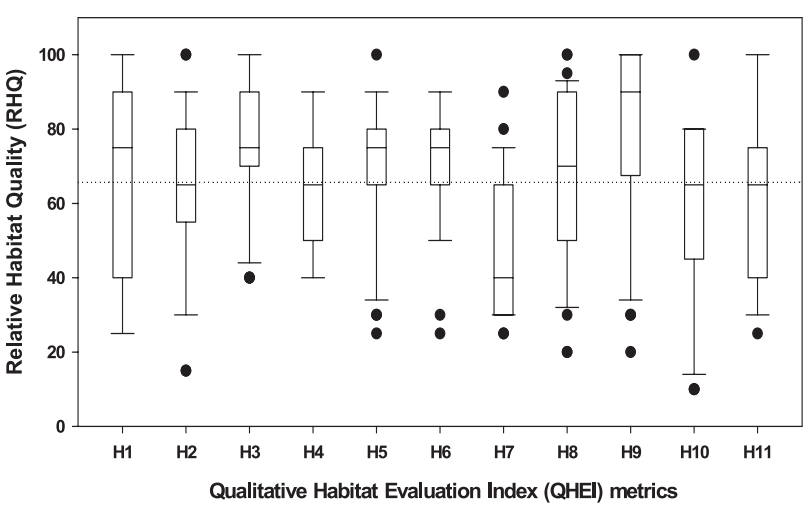

Fig. 4. Relative habitat quality (RHQ) of each parameter in Geum River Watershed; values were calculated as a proportion of each habitat metric, converting by maximum habitat metric values.

by channel modification and flow regime. The metric of H4 (channel flow status, 64.4\%) had low score in the headwater watershed and its tributaries due to reduced flows, small weirs in the channel, and an artificial reservoir such as Deacheong and Yongdam reservoirs. Metrics of H11 (dam construction, 60.2\%) showed relatively low variance.

The analysis of secondary habitat components (channel characteristics) showed that the metric scores of H5 (channel alteration, 68.2\%) were low due to urban development. Those sites were usually located in the urban stream and recreational resorts. The metric scores of H6 (bottom scouring/sediment deposition, 71.7\%), were significantly lower $(p<0.05)$ in the downstream than upstream due to rapid sedimentation processes caused by weir and dam, usually associated with flow reduction. The metric scores of $\mathrm{H} 7$ (frequency of riffles or bends) were the lowest (46.5\%) in the entire metrics except some sites, because we usually decide the site at bridges in the stream mainly showed lower channel sinuosity so far. Tertiary habitat components analyses showed that the metric scores of H8 (bank stability, $66.1 \%$ ), H9 (bank vegetative protection, $78 \%$ ), and H10 (riparian vegetative zone with, 58.2\%) were low in the urban streams, which is directly influenced by a modified channel, riverside roads, and recreational parks. These sites usually had unstable banks and artifacts by erosion and narrow riparian vegetative zone.

\section{Total Mercury Concentrations in the Sentinel Species}

Tissue analysis of liver, gill, kidney, vertebra, and muscle using a sentinel species of Zacco platypus at 33 streams and rivers showed that the mercury levels varied depending on the regional characteristics in the watershed. Contents of $\mathrm{Hg}$ in each tissue were converted to the relative proportion to compare differences among 5 tissues (Fig. 5). According to the tissue sample analysis, the mean level of $\mathrm{Hg}$ was the highest in muscle $(34.9 \%)$ and the lowest in gill (7.1\%). Previous studies

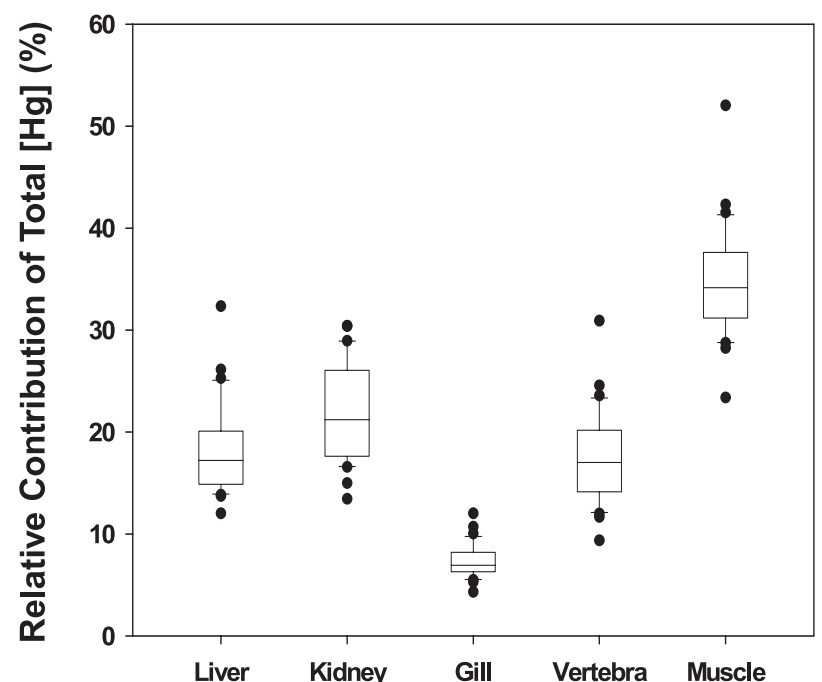

Fish Tissues

Fig. 5. Relative contribution of total $\mathrm{Hg}$ in various tissues of the sentinel species (Zacco platypus).

$[4,37-38]$ showed that muscle tissues had the highest mercury concentrations, as shown in our study. In the meantime, previous researchers [24, 39] reported highest levels in the kidney and liver tissues, so the pattern is not consistent in these studies. We found that there was no high correlation ([Hg] vs IBI, $r=0.371$, $p=0.034$ ) between total $\mathrm{Hg}$ and the ecological health condition of IBI values. However, high Hg contents were observed where IBI values were high. Therefore, $[\mathrm{Hg}]$ concentrations in fish tissue were not directly related with IBI value for ecological health assessments and QHEI values for habitat evaluations and chemical water quality [24]. In fact, $[\mathrm{Hg}]$ levels in our study did not affect fish individually when we compared the level with $[\mathrm{Hg}]$ standard of the U.S. EPA guideline. Hence, this $[\mathrm{Hg}]$ parameter could not respond with the evaluation method under the fish community level and standard water quality assessment.

\section{Fish Fauna and Composition}

A total of 12 families and 59 species were observed in 33 streams of the Geum River watershed. The most dominant families were Cyprinidae (37 species) and Cobitidae (4 species). Other families such as Bagridae, Siluridae, Amblycipitidae Osmeridae, Centropomidae, Centrarchidae, Odontobutidae, Gobiidae, Adrianichthyidae, and Channidae had 1 or 2 species (Table 3). The relative abundance of Cyprinidae was $>85 \%$ in the Geum River, and these results were in accordance with the previous studies of streams that flow in southwestern Korea [25]. Zacco platypus was the most abundant species, shoeing $27.6 \%$ relative abundance and $100 \%$ constancy. Zacco temminkii, Acheilognathus intermedia, and Pseudogobio esocinus were subdominant species with $>5.0 \%$ in 
Table 3. Fish fauna and guild compositions in Geum River Watershed.

\begin{tabular}{|c|c|c|c|c|c|}
\hline Species & Tol. G & Tro. G & TNI & RA $(\%)$ & Cons. $(\%)$ \\
\hline \multicolumn{6}{|c|}{ Cyprinidae } \\
\hline Cyprinus carpio & $\mathrm{TS}$ & $\mathrm{O}$ & 4 & 0.07 & 9.09 \\
\hline Carassius auratus & TS & $\mathrm{O}$ & 120 & 2.20 & 30.30 \\
\hline Carassius cuvieri & $\mathrm{TS}$ & $\mathrm{O}$ & 9 & 0.17 & 12.12 \\
\hline Rhodeus uyekii & IS & $\mathrm{O}$ & 52 & 0.95 & 24.24 \\
\hline Rhodeus notatus & IS & $\mathrm{O}$ & 57 & 1.05 & 15.15 \\
\hline Acheilognathus lanceolatus & SS & $\mathrm{O}$ & 421 & 7.73 & 60.61 \\
\hline Acheilognathus koreensis & IS & $\mathrm{O}$ & 124 & 2.28 & 24.24 \\
\hline Acheilognathus yamatsuatea & SS & $\mathrm{O}$ & 27 & 0.50 & 21.21 \\
\hline Acheilognathus rhombeus & IS & $\mathrm{O}$ & 3 & 0.06 & 9.09 \\
\hline Acanthorhodeus macropterus & IS & $\mathrm{O}$ & 12 & 0.22 & 18.18 \\
\hline Acanthorhodeus gracilis & IS & $\mathrm{O}$ & 86 & 1.58 & 24.24 \\
\hline Pseudorasbora parva & TS & $\mathrm{O}$ & 171 & 3.14 & 42.42 \\
\hline Pungtungia herzi & SS & I & 133 & 2.44 & 57.58 \\
\hline Pseudopungtungia nigra* & SS & I & 22 & 0.40 & 15.15 \\
\hline Coreoleuciscus splendidus & SS & I & 38 & 0.70 & 27.27 \\
\hline Sarcocheilichthys variegatus wakiyae & SS & I & 1 & 0.02 & 3.03 \\
\hline Sarcocheilichthys nigripinnis morii & IS & I & 22 & 0.40 & 18.18 \\
\hline Gnathopogon strigatus & IS & I & 164 & 3.01 & 45.45 \\
\hline Squalidus gracilis majimae & IS & I & 103 & 1.89 & 36.36 \\
\hline Squalidus japonicus coreanus & IS & $\mathrm{O}$ & 28 & 0.51 & 24.24 \\
\hline Hamibarbus labeo & $\mathrm{TS}$ & I & 80 & 1.47 & 39.39 \\
\hline Hamibarbus longirostris & SS & I & 135 & 2.48 & 63.64 \\
\hline Pseudogobio esocinus & IS & I & 309 & 5.67 & 84.85 \\
\hline Abbottina rivularis & IS & $\mathrm{O}$ & 6 & 0.11 & 9.09 \\
\hline Abbottina springeri & IS & $\mathrm{O}$ & 3 & 0.06 & 3.03 \\
\hline Gobiobotia macrocephala* & SS & I & 2 & 0.04 & 3.03 \\
\hline Gobiobotia brevibarba* & SS & I & 17 & 0.31 & 15.15 \\
\hline Gobiobotia nakdongensis* & SS & I & 23 & 0.42 & 3.03 \\
\hline Microphysogobio yaluensis & IS & $\mathrm{O}$ & 196 & 3.60 & 63.64 \\
\hline Microphysogobio jeoni & IS & I & 10 & 0.18 & 9.09 \\
\hline Rhynchocypris oxycephalus & SS & I & 50 & 0.92 & 6.06 \\
\hline Aphyocypris chinensis & $\mathrm{TS}$ & $\mathrm{O}$ & 1 & 0.02 & 3.03 \\
\hline Zacco temminckii & SS & I & 522 & 9.58 & 39.39 \\
\hline Zacco platypus & $\mathrm{TS}$ & $\mathrm{O}$ & 1,503 & 27.58 & 100 \\
\hline Opsarichthys uncirostris amurensis & IS & $\mathrm{C}$ & 262 & 4.81 & 57.58 \\
\hline Squaliobarbus curriculus & IS & $\mathrm{O}$ & 20 & 0.37 & 15.15 \\
\hline Hemiculter eigenmanni & TS & $\mathrm{O}$ & 6 & 0.11 & 3.03 \\
\hline
\end{tabular}


Table 3. Continued

\begin{tabular}{|c|c|c|c|c|c|}
\hline \multicolumn{6}{|c|}{ Cobitidae } \\
\hline Misgurnus anguillicaudatus & TS & $\mathrm{H}$ & 57 & 1.05 & 39.39 \\
\hline Misgurnus mizolepis & TS & $\mathrm{H}$ & 40 & 0.73 & 45.45 \\
\hline Iksookimia koreensis & SS & I & 27 & 0.50 & 30.30 \\
\hline Cobitis lutheri & IS & I & 1 & 0.02 & 3.03 \\
\hline \multicolumn{6}{|c|}{ Bagridae } \\
\hline Pseudobagrus fulvidraco & IS & $\mathrm{C}$ & 5 & 0.09 & 6.06 \\
\hline Pseudobagrus koreanus & SS & I & 47 & 0.86 & 27.27 \\
\hline \multicolumn{6}{|c|}{ Siluridae } \\
\hline Silurus asotus & TS & $\mathrm{C}$ & 10 & 0.18 & 15.15 \\
\hline Silurus microdorsalis & SS & $\mathrm{C}$ & 2 & 0.04 & 3.03 \\
\hline \multicolumn{6}{|c|}{ Amblycipitidae } \\
\hline Liobagrus mediadiposalis & SS & I & 13 & 0.24 & 9.09 \\
\hline Liobagrus obesus* & SS & I & 1 & 0.02 & 3.03 \\
\hline \multicolumn{6}{|c|}{ Osmeridae } \\
\hline Hypomesus nipponensis & SS & $\mathrm{H}$ & 2 & 0.04 & 3.03 \\
\hline Plecoglossus altivelis altivelis & SS & $\mathrm{H}$ & 1 & 0.02 & 3.03 \\
\hline \multicolumn{6}{|c|}{ Adrianichthyidae } \\
\hline Oryzias sinensis & TS & $\mathrm{O}$ & 5 & 0.09 & 3.03 \\
\hline \multicolumn{6}{|c|}{ Centropomidae } \\
\hline Siniperca scherzeri & SS & $\mathrm{C}$ & 2 & 0.04 & 6.06 \\
\hline Coreoperca herzi & SS & $\mathrm{C}$ & 16 & 0.29 & 21.21 \\
\hline \multicolumn{6}{|c|}{ Centrarchidae } \\
\hline Lepomis macrochirus $\dagger$ & TS & $\mathrm{I}$ & 3 & 0.06 & 6.06 \\
\hline Micropterus salmoides $\dagger$ & TS & $\mathrm{C}$ & 27 & 0.50 & 27.27 \\
\hline \multicolumn{6}{|c|}{ Odontobutidae } \\
\hline Odontobutis platycephala & SS & $\mathrm{C}$ & 57 & 1.05 & 39.39 \\
\hline Odontobutis interrupta & IS & $\mathrm{C}$ & 164 & 3.01 & 75.76 \\
\hline \multicolumn{6}{|c|}{ Gobiidae } \\
\hline Rhinogobius brunneus & IS & I & 179 & 3.29 & 57.58 \\
\hline Tridentiger brevispinis & IS & I & 47 & 0.86 & 24.24 \\
\hline \multicolumn{6}{|c|}{ Channidae } \\
\hline Channa argus & $\mathrm{TS}$ & $\mathrm{C}$ & 1 & 0.02 & 3.03 \\
\hline
\end{tabular}

$*=$ endangered species, $\dagger=$ Exotic species Tol. $\mathrm{G}=$ Tolerance Guild, Tro. $\mathrm{G}=$ Trophic Guild, $\mathrm{TNI}=$ Total number of individuals, $\mathrm{RA}=$ Relative abundance, Cons. $=$ Constancy, $\mathrm{TS}=$ Tolerent species, $\mathrm{SS}=$ Sensitive species, IS $=$ Intermediate species,

$\mathrm{O}=$ Omnivores, $\mathrm{I}=$ Insectivores, $\mathrm{C}=$ Carnivores, $\mathrm{H}=$ Herbivores

relative abundance (Table 3). The endangered species observed were 5 species such as Pseudopungtungia nigra, Liobagrus obesus, Gobiobotia nakdongensis, Gobiobotia macrocephala, and Gobiobotia brevibarba, and the relative abundance was $0.5 \%$ in the study. These species were limitedly inhabited with good condition of physical habitat and water quality $(\mathrm{BOD}<1)$ so that they needed more effort to maintain habitat condition and to preserve and manage continuously. The number of exotic invasive species observed in the sampling were 3, and the species were Micropterus salmoides, Lepomis macrochirus, and Carassius cuvieri. However, the actual 
number inhabiting the site could be underestimated due to limited sampling gear and time.

The relative proportion of sensitive species that represented qualitative degradation of water quality and contamination was 30\% (1579 individuals), and tolerant species were $37.4 \% \quad(2,037$ individuals). Intermediate species that were not sensitively impacted by water pollution were $33.6 \%$ (1,833 individuals). Thus, proportion analysis among tolerance guilds showed that tolerant species were slightly dominated, but there were not too many differences among the species and sampling sites. Correlation analysis (Fig. 6) between tolerance guilds and other parameters (IBI, QHEI, and BOD) were seen to be significant $(p<0.05)$ among all parameters we used. Especially, sensitive species had negative functional relationships with BOD $(r=-0.715$, $p<0.001)$ and positive with IBI $(r=0.683, p<0.001)$ and QHEI $(r=0.573, p=0.001)$.
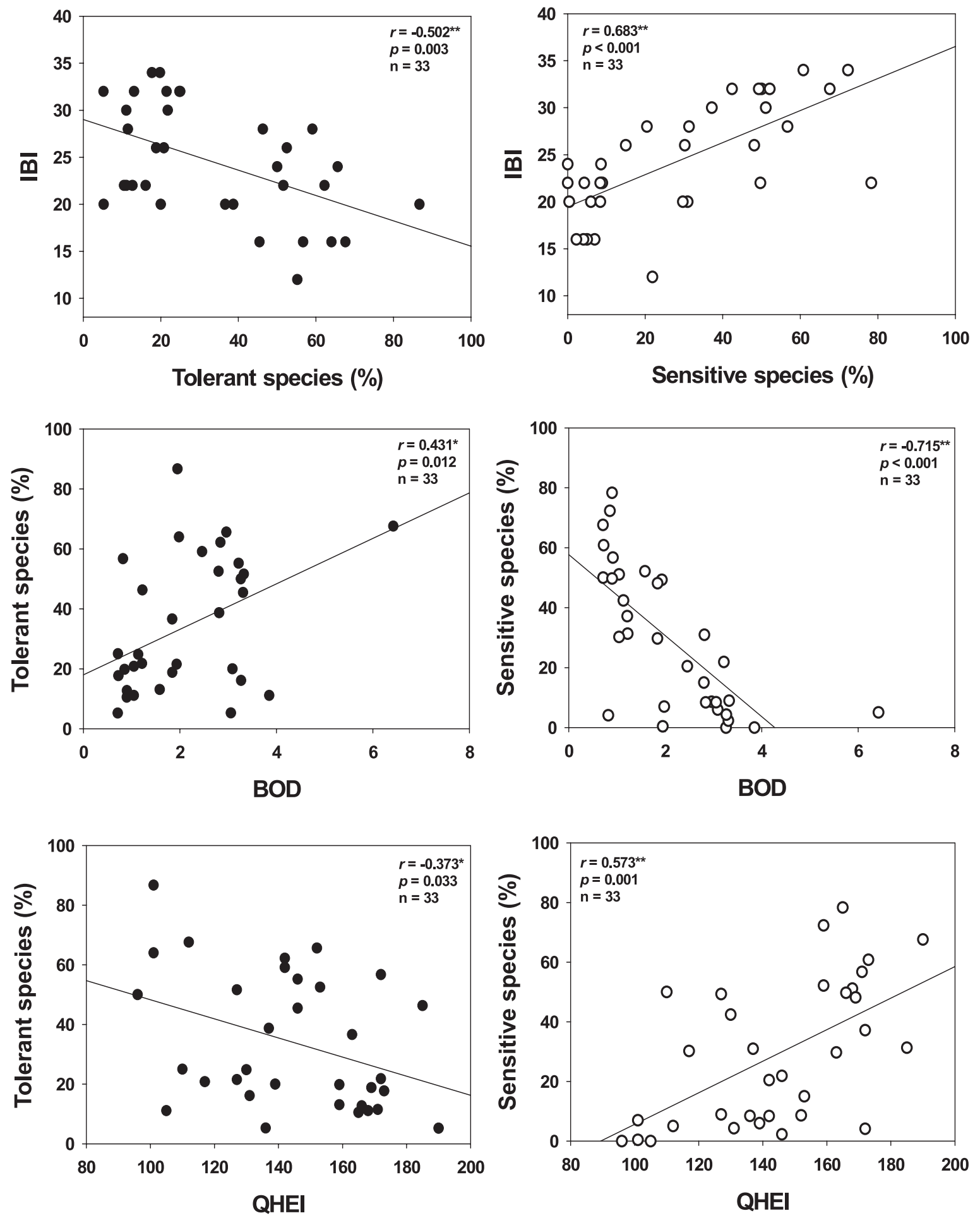

Fig. 6. Regression analyses and their relations in the biological, chemical, and physical parameters. 
Fish Trophic Guilds

Omnivore fish that can diet on all types of food tended to increase with physiochemical degradation of habitat and water quality, whereas the insectivores, mostly dieting on aquatic macroinvertebrates, decreased with chemical degradation. Thus, the dominance of omnivores was evident and this was caused by Zacco platypus, the most dominant species (over 50\% in entire omnivore) in the sampling. Correlation analysis (Fig. 7) was conducted between trophic guilds and other parameters (IBI, QHEI, and BOD). The proportion of omnivores did not show any statistical significance $(p>0.05)$ with other parameters but tended to increase
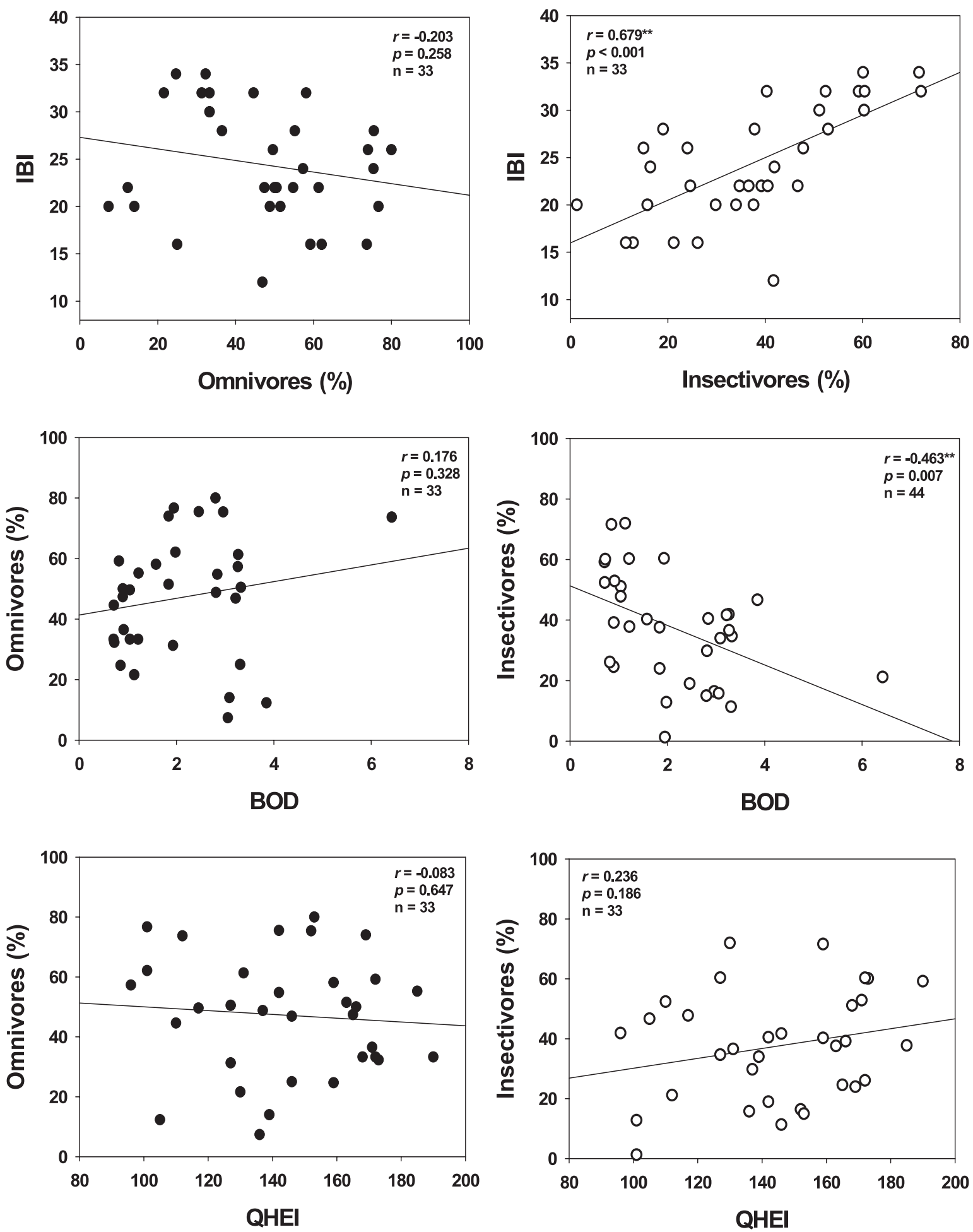

Fig. 7. Regression analysis of the multimetic IBI values and fish guild parameters on chemical water quality, feeding types, and habitat conditions. 


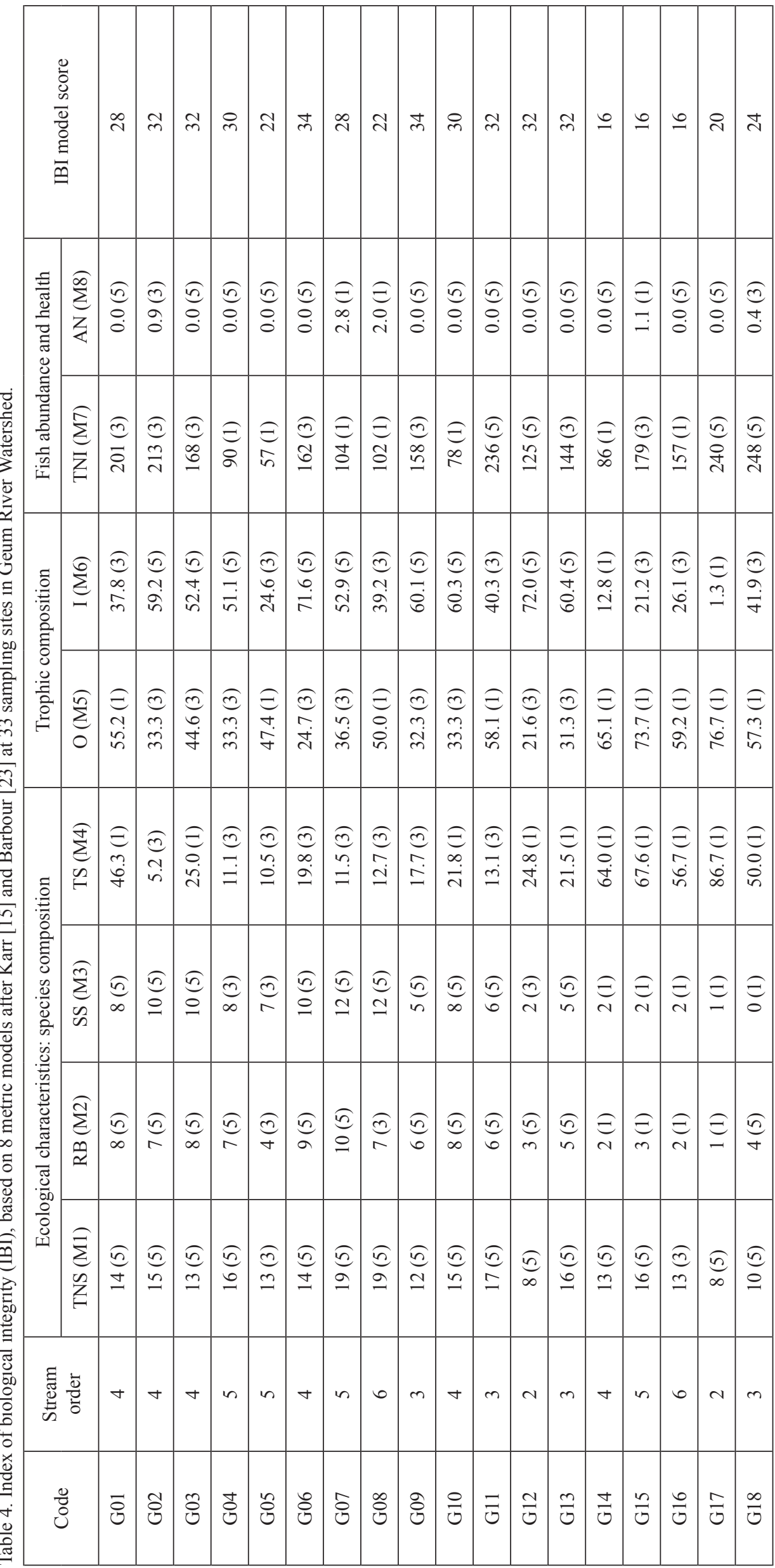




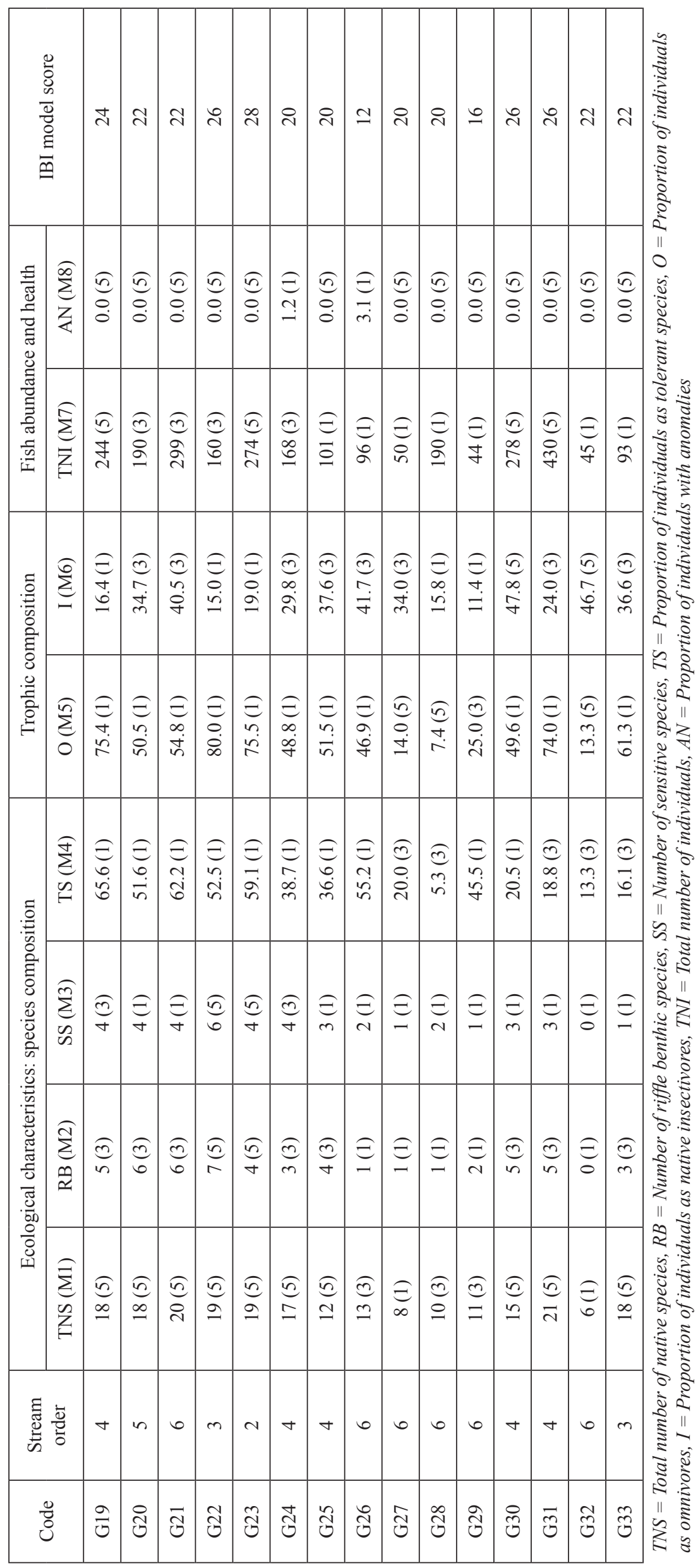


by habitat disturbance based on QHEI and BOD degradation. However, insectivores can reflect the improvement of biological integrity (IBI, $r=0.679$, $p<0.001)$ and sensitively respond with deterioration of
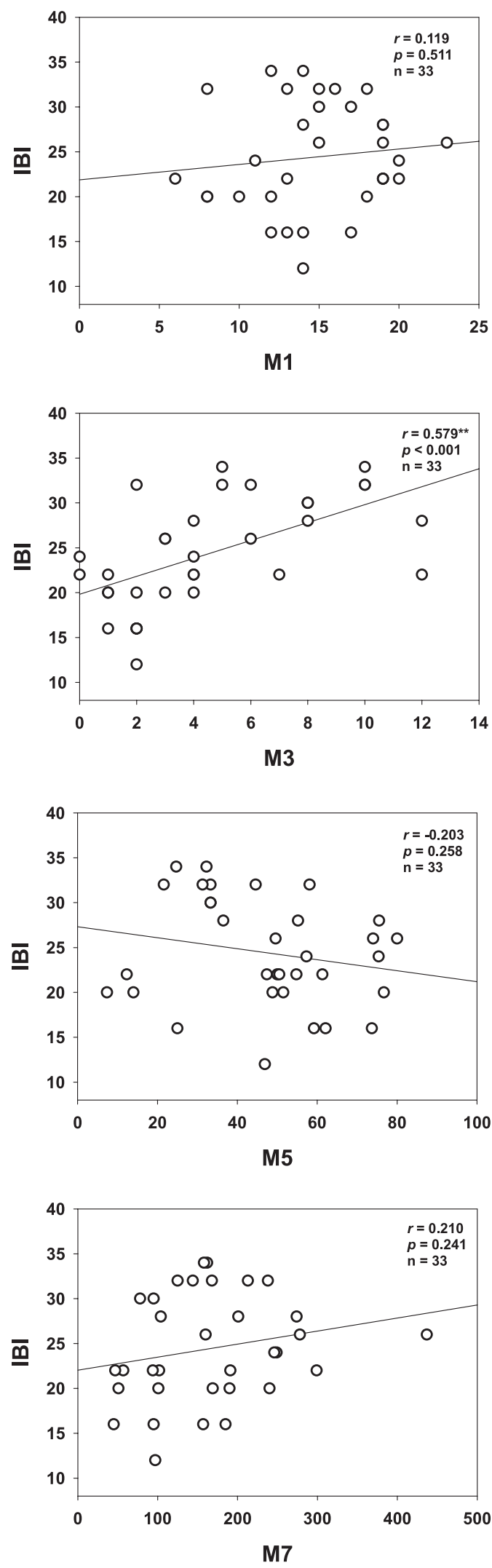

water quality (BOD, $r=-0.463, p=0.007$ ). Therefore, aquatic macroinvertebrates, major diet sources of insectivores, usually depended on organic matters as their food, and this organic matter reacted as an
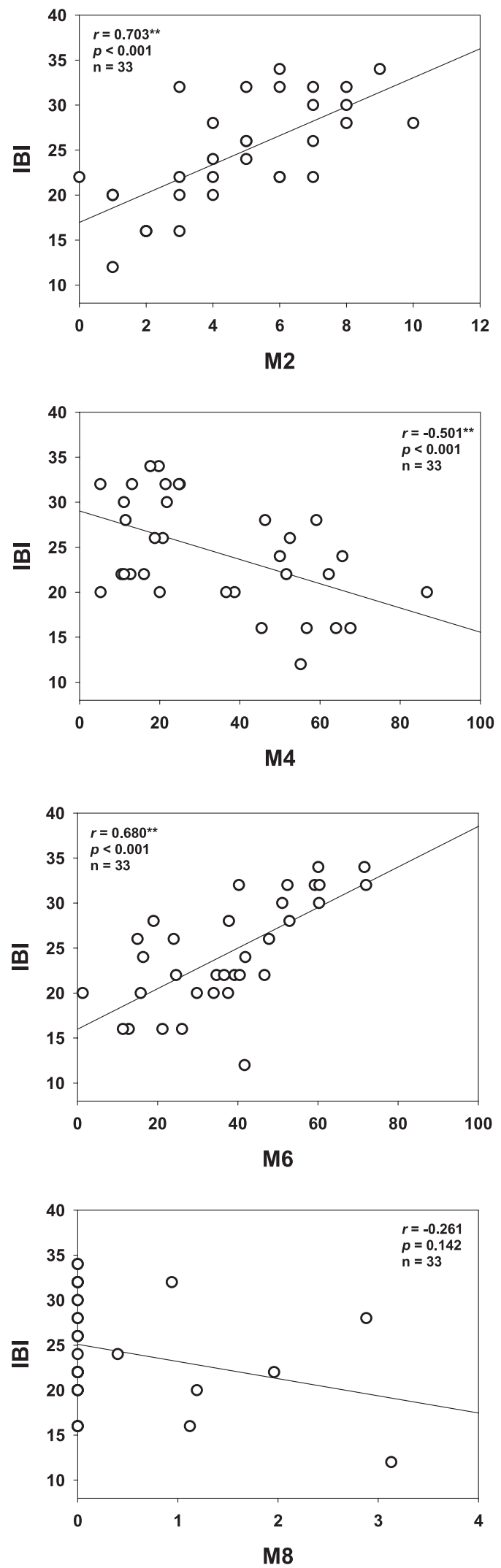

Fig. 8. Relations of the index of biological integrity (IBI) to each fish model metric. 
Table 5. Comparison of prediction results with different input variables.

\begin{tabular}{|c|c|c|c|c|c|c|}
\hline \multirow{2}{*}{ Input variables } & \multirow{2}{*}{ Epoch \# (Run \#) } & \multirow{2}{*}{ MLP model } & \multicolumn{2}{|c|}{ Training } & \multicolumn{2}{|c|}{ Testing } \\
\cline { 4 - 7 } & & MSE & $\mathrm{R}_{2}$ & MSE $^{2}$ & $\mathrm{R}_{2}$ \\
\hline 1 input (QHEI) & $1000(3)$ & MLP 1-3-1 & 21.854 & 0.235 & 76.452 & 0.128 \\
\hline 4 input (BOD, TSS, EC, TP) & $1000(3)$ & MLP 4-4-1 & 6.646 & 0.769 & 64.852 & 0.029 \\
\hline 5 input (QHEI, BOD, TSS, EC, TP) & $2000(3)$ & MLP 5-5-1 & 0.092 & 0.997 & 127.263 & 0.107 \\
\hline 14 input (all variables) & $500(3)$ & MLP 14-5-1 & 0.016 & 0.999 & 24.423 & 0.894 \\
\hline
\end{tabular}

environmental factor affecting populations of aquatic macroinvertebrates.

\section{Biological Integrity}

Values of IBI based on the 8-metrics fish model averaged 24.4 (range: 12-34, $\mathrm{n}=33$ ) and were categorized as in fair condition (Table 4). In this study, excellent streams were not found. However, 5 of 6 streams in the Gap and Mi-ho stream, passing through an urban area (Daejeon and Cheongju), were judged to be in fair condition (Fig. 8). Thus, stream channelization and nutrient enrichments directly influenced the stream degradations in the urban area [24]. Thirteen streams located in the lowland area were categorized as being in fair-poor condition. Though there were some longitudinal differences in the watershed, physical habitat and biological conditions in the up-region were maintained well and chemical pollution was rare. In contrast, in the mid-region the urban environment largely impacted the river due to continuous effluents of wastewater disposal plants and industrial complex, resulting in habitat simplification with sand sedimentation. These degradations also influenced the lower region of the river as shown in the degradation of biological health. Among eight metrics used to calculate the IBI score, values of the M4 (proportion of individuals as tolerant species) and M5 (proportion of individuals as omnivores) were relatively low $($ mean $<2)$ because of dominance of tolerant species and omnivorous species in the fish community. In the meantime, mean values of M1 (total number of native species) and M8 (proportion of individuals with anomalies) were $>4$, indicating good condition (Table 4). The correlation analysis between IBI scores and each metric indicated that IBI scores increased with decreases of tolerant species, omnivore species, and abnormal fish species. Our results agree with previous results of integrative health modeling [19]. Especially the metric model values of M2, M3, M4, and M6 had high significant relationships and were highly significant $(p<0.001)$ with biological health, based on the IBI scores. These results suggest that ecological health, based on the model values, is closely influenced by the physical habitat conditions and chemical regimes (Fig. 9).

\section{IBI Value Prediction Using the Approach of Artificial Neural Network}

Model values of IBI were predicted by 14 variables of biological, physical, and chemical factor characteristics
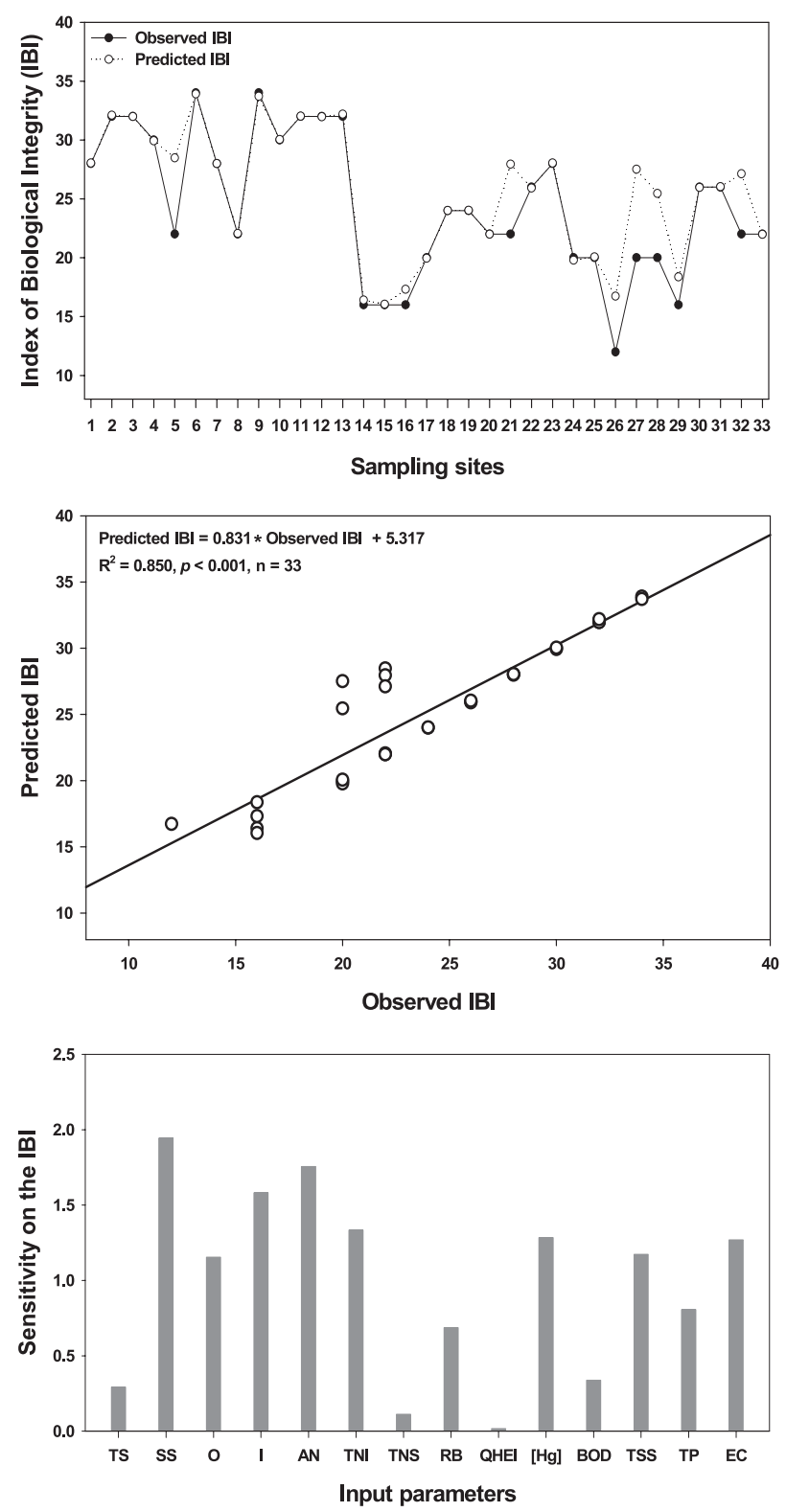

Fig. 9. Output of artificial neural network modeling in Geum River Watershed. 
1 input (QHEI)

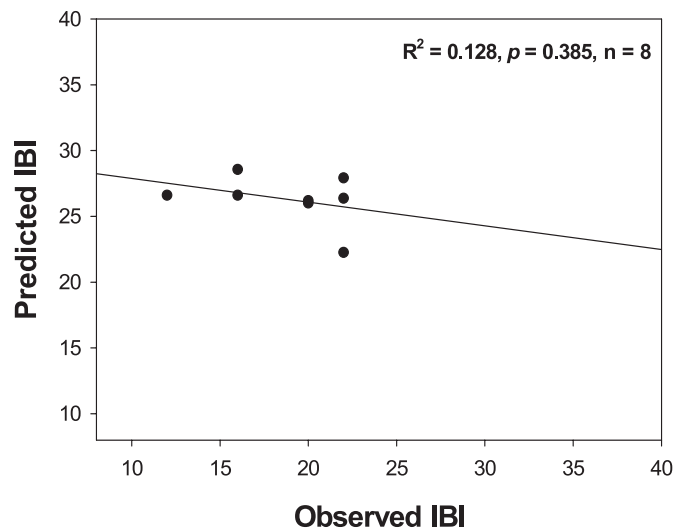

5 input (QHEI, BOD, TSS, EC, TP)

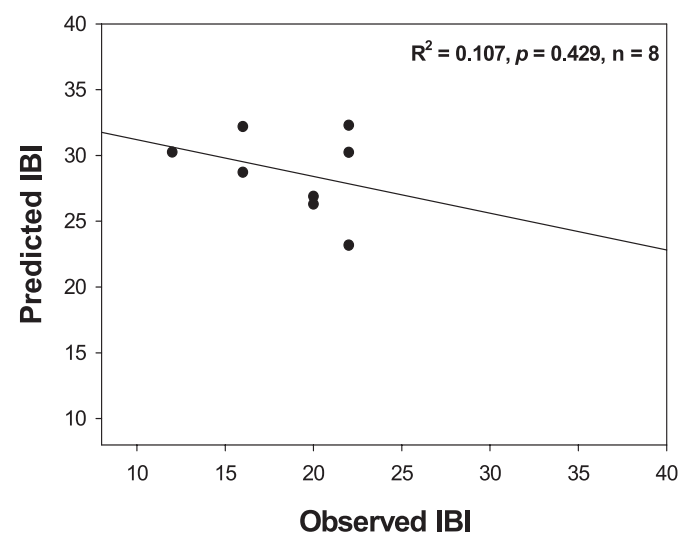

4 input (BOD, TSS, EC, TP)

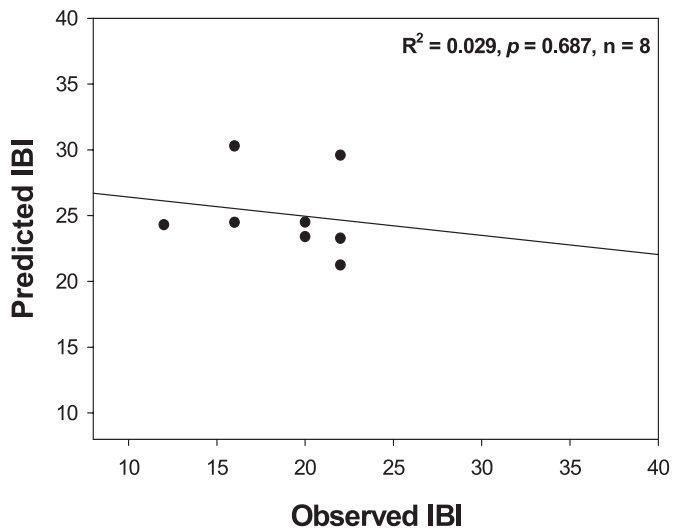

14 input (all variables)

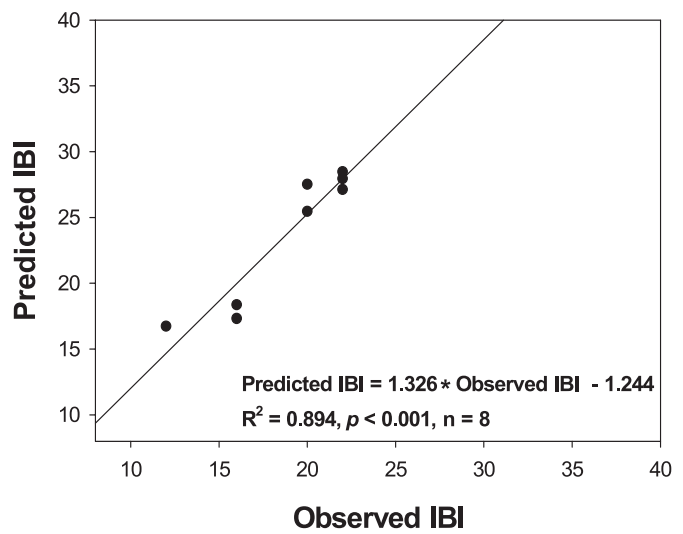

Fig. 10. Output of testing results with various input variables.

used by an artificial neural network (ANN) model. For the ANN model, the MLP 14-5-1 model was determined (minimum MSE $=0.000078$ ). Generally, the number of hidden layers was not mainly affected to the accuracy in the compositions of the neural network, and the existence of the hidden layer provided enough accuracy. Moreover, in the case of more than 4 nodes in the hidden layer, it showed sufficient accuracy regardless of the node number in the input layer.

Analysis of the MLP 141-5-1 model showed that the observed IBI values were highly correlated with predicted IBI values in the training sites $\left(\mathrm{R}^{2}=0.999, \mathrm{MSE}=0.0156\right)$ and testing sites $\left(\mathrm{R}^{2}=0.894\right.$, MSE = 27.4227), indicating a high-efficiency MLP model (Table 5). Also, the predicting model for Geum River predicted the actual IBI values (predicted IBI $=0.831 *$ Observed IBI +5.317$)$, and showed significantly high predictability $\left(\mathrm{R}^{2}=0.850, p<0.001, \mathrm{n}\right.$ $=33$ ). From the results of the sensitivity analysis (input by $\pm 1 \mathrm{SD}$ ) in this model, input variables of sensitive species (SS), insectivores (I), and anomalies (AN) among biological categories showed high sensitivity with IBI, but TNS and QHEI were less affected by the IBI prediction (Fig. 9). In the variance analysis of input variables, the model of MLP 1-3-1 model showed the least predictability when it was only applied for physical variable (QHEI). In contrast, the MLP 4-4-1 model using chemical variables of BOD, TSS, EC, and TP and the MLP 5-5-1 model using physicochemical variables of QHEI, BOD, TSS, EC, and TP) showed greater predictability $\left(\mathrm{R}^{2}>0.70\right)$ in the training data but was less predictable $\left(\mathrm{R}^{2}<0.11\right)$ in testing data. The MLP 14-5-1 model applied all available 14 input variables showing great predictability. Thus, it may be difficult to predict accurately integrated health condition applied by physicochemical environmental factors only, regardless of biological factors (Table 5, Fig. 10).

\section{Statistical Relationships of Various Parameters}

PCA (SPSS 2004) using various factors was conducted to diagnose the effects of variables such as on-stream components. For diagnosing the interrelationship among dependent factors, we used the "direct oblimin rotation" method $(\delta=0.8)$ in the "oblique rotation" method and decided two factors (eigen value: 1.0). As a result, Factor 1 (SS, BOD, IBI, QHEI) could be described with 55.4\% and Factor 2 (TS, O, I) with 17.9\%. Major influence components in the PCA were SS (0.914, Factor 1), BOD (-0.848, Factor 1), and TS (0.864, Factor 2) (Table 6). 
Table 6. Principal component analysis (PCA) using biological (IBI, tolerance, and trophic guilds), physical (QHEI), and chemical parameters (BOD).

\begin{tabular}{|c|c|c|c|}
\hline \multicolumn{4}{|c|}{ Principal component analysis / Direct oblimin rotation } \\
$(\boldsymbol{\delta}=\mathbf{0 . 8})$ / Eigenvalue 1.0 \\
\hline $\begin{array}{c}\text { Structure } \\
\text { matrix }\end{array}$ & Factor 1 & Factor 2 & $h 2$ \\
\hline SS & $\mathbf{0 . 9 1 4}$ & -0.611 & 0.839 \\
\hline BOD & $\mathbf{- 0 . 8 4 8}$ & 0.452 & 0.729 \\
\hline IBI & $\mathbf{0 . 7 8 9}$ & -0.603 & 0.643 \\
\hline QHEI & $\mathbf{0 . 7 1 4}$ & -0.237 & 0.578 \\
\hline TS & -0.634 & $\mathbf{0 . 8 6 4}$ & 0.763 \\
\hline O & -0.230 & $\mathbf{0 . 8 4 1}$ & 0.845 \\
\hline I & 0.653 & $\mathbf{- 0 . 8 3 7}$ & 0.730 \\
\hline Eigenvalue & 3.875 & 1.252 & \\
\hline $\begin{array}{c}\text { Proportion of } \\
\text { variance }\end{array}$ & $\mathbf{5 5 . 4}$ & $\mathbf{1 7 . 9}$ & \\
\hline $\begin{array}{c}\text { Cumulative } \\
\text { proportion }\end{array}$ & 55.4 & 73.3 & \\
\hline
\end{tabular}

$I B I=$ Index of biological integrity, $Q H E I=$ Qualitative habitat evaluation index, $B O D=$ Biological oxygen demand, $S S=$ Proportion of individuals as sensitive species, $T S=$ Proportion of individuals as tolerant species, $O=$ Proportion of individuals as omnivores, $I=$ Proportion of individuals as native insectivores

Hierarchical cluster analysis [36] showed that the watershed streams were categorized into four groups (Fig. 11). Group I showed Lower IBI in spite of good habitat conditions and Groups II and III showed intermediate characteristics. Group II usually was located in the upstream reach and maintained relatively good habitat condition and water quality. There also appeared a high ratio of sensitive and insectivore species. In contrast, Group III was usually located in middle and downstream reaches and showed a relatively high proportion of tolerant and omnivore species. Group IV was located downstream in the mainstream with an extremely high ratio of intermediate species in tolerance guild (Fig. 11). The plotting analysis were conducted between two factors in PCA (Table 6) and four groups in cluster analysis (Fig. 11) to diagnose how physical habitat and chemical parameters affect each group (PCOrd). Group II was often affected by physical habitat parameters. Especially Group I indicated good overall habitat conditions mainly affected by $\mathrm{H} 1$ and $\mathrm{H} 2$ among the habitat metrics, indicating substrate structure and natural habitat condition but varied with water quality condition. Group III was mainly affected by chemical water quality parameters, especially organic pollution indicator (BOD). In contract, it seems to be varied as water quality changes by various pollution sources and habitat specificity by the variation of the middle and downstream reaches (Fig. 12).

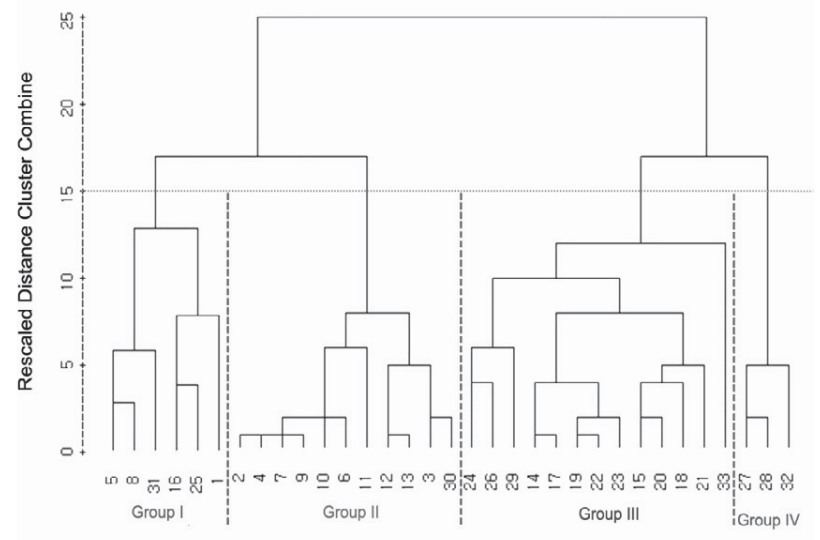

Fig. 11. Dendrogram of Geum-River Watershed sites based on hierarchical cluster analysis.

Overall, our results suggest that consistent stream/ river conservations are required in the upstream and downstream regions with low IBI values and low QHEI. The degradations were mainly due to effluents from the point sources of wastewater disposal plants and industrial complex in urban midstream and downstream.

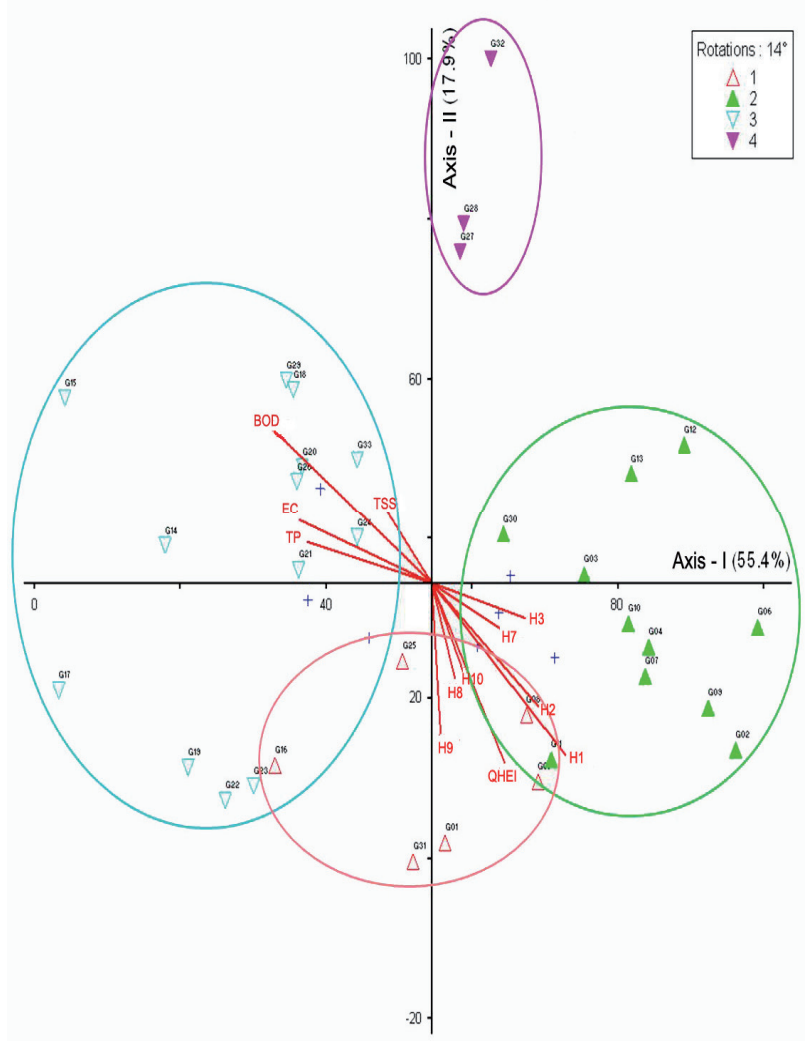

Fig. 12. PCA of Geum River Watershed based on physical habitat, chemical condition, and biological components; abbreviations are: $\mathrm{G} 01 \sim \mathrm{G} 33$ = sampling sites, $\mathrm{QHEI}=$ Qualitative Habitat Evaluation Index, H1 H11 = habitat metrics, BOD = biological oxygen demand, TSS $=$ total suspended solids, $\mathrm{EC}=$ electric conductivity, $\mathrm{TP}=$ total phosphorus. 
So the habitat disturbance caused by artificial channel modification and organic matter pollution should be managed for restoration by the government.

\section{References}

1. DANIEL R.O., PAUL W., EUGENE A.S., LEV Y.Y., ELENA L. Nitrogen and phosphorus colimitation of phytoplankton in Lake Baikal: Insights from a spatial survey and nutrient enrichment experiments. Limnology and Oceanography. 62 (4), 1383, 2017.

2. MAGILLIGAN F.J., NISLOW K.H., KYNARD B.E., HACKMAN A.M. Immediate changes in stream channel geomorphology, aquatic habitat, and fish assemblages following dam removal in a small upland catchment. Geomorphology 252 (1), 158, 2016.

3. SILVIA V.M., SYLVAIN D., ADRIANO S.M. Substrate heterogeneity influences the trait composition of stream insect communities: an experimental in situ study. Freshwater science 35 (4), 1321, 2016.

4. BOEHLER S., STRECKER R., HEINRICH P., PROCHAZKA E., NORTHCOTT G.L., ATARIA J.M., TREMBLAY L.A. Assessment of urban stream sediment pollutants entering estuaries using chemical analysis and multiple bioassays to characterise biological activities. Science of The Total Environment, 593, 498, 2017.

5. PAVEL R., PETR V., HANA P., DANIEL F. Estimated Contribution of Selected Non-point Pollution Sources to the Phosphorus and Nitrogen Loads in Water Bodies of the Vltava River Basin. Soil \& Water Res. 11 (3) 196, 2016.

6. BOUCHARD R.W., NIEMELA S., GENET J.A., YODER C.O., SANDBERG J., CHARHART J.W., HELWIG D. A novel approach for the development of tiered use biological criteria for rivers and streams in an ecologically diverse landscape. Environmental monitoring and assessment, 188 (3), 196, 2016.

7. CARTER S.K., CARR N.B., FLATHER C.H., FLEISHMAN E., LEU M., NOON B.R., WOOD D.J. Assessing Ecological Integrity Using Multiscale Information from Bureau of Land Management Assessment and Monitoring Programs. 39, 2017.

8. NESTLER J.M., BAIG N,C., MADDOCK I. Achieving the aquatic ecosystem perspective: integrating interdisciplinary approaches to describe instream ecohydraulic processes. River Science: Research and Management for the $21^{\text {st }}$ Century, 84, 2016.

9. CARLISLE D.M., NELSON S.M., MAY J. Associations of stream health with altered flow and water temperature in the Sierra Nevada, California. Ecohydrology, 9 (6), 930, 2016.

10. FL VIO H.M., FERREIRA P., FORMIGO N., SVENDSEN J.C. Reconciling agriculture and stream restoration in Europe: A review relating to the EU Water Framework Directive. Science of the Total Environment, 596, 378, 2017.

11. MAIRE A., LAFFAILLE P., MAIRE J.F., BUISSON L. Identification of Priority Areas for the Conservation of Stream Fish Assemblages: Implications for River Management in France. River Research and Applications, 33 (4), 524, 2017.

12. SOAR P.J., WALLERSTEIN N.P., THORNE C.R. Quantifying river channel stability at the basin scale. Water, 9 (2), 133, 2017.
13. FL VIO H.M., FERREIRA P., FORMIGO N., SVENDSEN J.C. Reconciling agriculture and stream restoration in Europe: A review relating to the EU Water Framework Directive. Science of the Total Environment, 596, 378, 2017.

14. TULlOS D.D., PENROSE D.L., JENNINGS G.D. Development and application of a bioindicator for benthic habitat enhancement in the North Carolina Piedmont. Ecological Engineering, 27, 228, 2006.

15. WALTON B.M., SALLING M., WYLES J., WOLIN J. Biological integrity in urban streams: Toward resolving multiple dimensions of urbanization. Landscape and Urban Plan 79, 110, 2007.

16. HASSELMAN D.J., ARGO E.E., MCBRIDE M.C., BENTZEN P., SCHULTZ T.F., PEREZ-UMPHREY A.A., PALKOVACS E.P. Human disturbance causes the formation of a hybrid swarm between two naturally sympatric fish species. Molecular ecology, 23 (5), 1137, 2014.

17. AN K.G., LEE J.Y., BAE D.Y., KIM J.H., HWANG S.J., WON D.H., LEE J.K., KIM C.S. Ecological assessments of aquatic environment using multi-metric model in major nationwide stream watersheds. J. of Korean Society on Water Quality, 22, 796, 2006.

18. LEE J.H., AN K.G. Integrative restoration assessment of an urban stream using multiple modeling approaches with physical, chemical, and biological integrity indicators. Ecological engineering, 62, 153, 2014.

19. PIPPY B.A., KIDD K.A., MUNKITTRICK K.R., MERCER A., HUNT H. Use of the Atlantic nut clam (Nucula proxima) and catworm (Nephtys incisa) in a sentinel species approach for monitoring the health of Bay of Fundy estuaries. Marine pollution bulletin, 106 (1), 225, 2016.

20. MACEDO D.R., HUGHES R.M., FERREIRA W.R., FIRMIANO K.R., SILVA D.R., LIGEIRO R., CALLISTO M. Development of a benthic macroinvertebrate multimetric index (MMI) for Neotropical Savanna headwater streams. Ecological Indicators, 64, 132, 2016.

21. MONCAYO-ESTRADA R., LYONS J., RAMIREZHERREJON J.P., ESCALERA-GALLARDO C., CAMPOS-CAMPOS O. Status and Trends in Biotic Integrity in a Sub-Tropical River Drainage: Analysis of the Fish Assemblage Over a Three Decade Period. River research and applications, 31 (7), 808, 2015.

22. KRAUSE J.R., BERTRAND K.N., KAFLE A., TROELSTRUP N.H. A fish index of biotic integrity for South Dakota's Northern Glaciated Plains Ecoregion. Ecological indicators, 34, 313, 2013.

23. SHEN Q., YU J.J., CHEN H., HU J.X., CHEN M.X., ZHEN J.X., LI D., ZHANG J.F. Planktonic index of biotic integrity (P-IBI) for water source assessment. J. Hydroecol., 23 (2), 26, 2012 [In Chinese].

24. LEE E.H., YOON S.H., LEE J.H., AN K.G. Total Mercury contents in the Tissues of Zacco platypus and Ecological Health Assessments in Association with Stream Habitat Characteristics. Korean J. of Limnology, 41, 188, 2008.

25. BAE E., JUNG J. Prediction of Shift in Fish Distributions in the Geum River Watershed under Climate Change. Ecology and Resilient Infrastructure, 2 (3), 198, 2015.

26. KIM J.H., YOON J.D., BAEK S.H., PARK S.H., LEE J.W., LEE J.A., JANG M.H. An efficiency analysis of a naturelike fishway for freshwater fish ascending a large Korean river. Water, 8 (1), 3, 2015.

27. CHOI J.W., LEE E.H., LEE J.H., AN K.G. Biological Water Quality Assessments Using Fish Assemblage 
in Nakdong River Watershed. Korean J. of Limnology, 40, 254, 2007.

28. KWAK S.D., CHOI J.W., AN K.G. Chemical Water Quality and Fish Component Analyses in the Periods of Before-and After-the Weir Constructions in Yeongsan River. Journal of Ecology and Environment, 39 (1), 99, 2016.

29. BUSS D.F., CARLISLE D.M., CHON T.S., CULP J., HARDING J.S., KEIZER-VLEK H.E., HUGHES R.M. Stream biomonitoring using macroinvertebrates around the globe: a comparison of large-scale programs. Environmental monitoring and assessment, 187 (1), 4132, 2015.

30. SHI Z.H., HUANG X.D., AI L., FANG N.F., WU G.L. Quantitative analysis of factors controlling sediment yield in mountainous watersheds. Geomorphology, 226, 193, 014.

31. SIMON T.P., BURSKEY J.L. Deformity, Erosion, Lesion, and Tumor Occurrence, Fluctuating Asymmetry, and Population Parameters for Bluntnose Minnow (Pimephales notatus) as Indicators of Recovering Water Quality in a Great Lakes Area of Concern, USA. Archives of environmental contamination and toxicology, 70 (2), 181-, 2016.

32. HATAMI R., PAUL W., SOOFIANI N.M., ASADOLLAH $\mathrm{S}$. Rapid bioassessment of macroinvertebrate communities is suitable for monitoring the impacts of fish farm effluents. Aquaculture, 468, 19, 2017.
33. GSCHWEND P.M. Environmental organic chemistry. John Wiley \& Sons. 2016.

34. CALDERON,M.S., AN K.G. An influence of mesohabitat structures (pool, riffle, and run) and land-use pattern on the index of biological integrity in the Geum River watershed. Journal of Ecology and Environment, 40 (1), 13, 2016.

35. HUANG J., GAO J. An ensemble simulation approach for artificial neural network: An example from chlorophyll a simulation in Lake Poyang, China. Ecological Informatics, 37, 52, 2017.

36. SPSS. SPSS systems for windows. Version $12.0 \mathrm{KO}$ for windows, USA. 2004

37. ASTANI E., VAHEDPOUR M., BABAEI H. Organic and Total Mercury Concentration in Fish Muscle and Thermodynamic Study of Organic Mercury Extraction in Fish Protein. ECOPERSIA, 4 (3), 1517, 2016.

38. ENDO T., OGASAWARA H., HAYASAKA M., HOTTA Y., KIMURA O., PETZKE K.J. Correlations between mercury concentration, and stable isotope ratios of carbon and nitrogen of amino acids in scalp hair from whale meat eaters and heavy fish eaters. Rapid Communications in Mass Spectrometry, 31 (9), 745, 2017.

39. KIM M.K., LEE Y.M., ZOH K.D. Spatial and temporal variation of total mercury and methylmercury in lacustrine wetland in Korea. Environmental Science and Pollution Research, 22 (9), 6578, 2015. 
